Emulating the Fast-Start Swimming Performance of the Chain Pickerel (Esox niger) Using a Mechanical Fish Design

by

Matthew Nicholas Watts

B.S., Physical Oceanography

United States Naval Academy, 2004

Submitted in Partial Fulfillment of the Requirements for the Degree of

Master of Science in Oceanographic Engineering

at the

Massachusetts Institute of Technology

and the

Woods Hole Oceanographic Institution

September 2006

(c) Matthew Nicholas Watts, 2006. All rights reserved.

The author hereby grants to MIT and WHOI permission to reproduce and to distribute publicly paper and electronic copies of this thesis document in whole or in part in any medium known or hereafter created.

Signature of Author

Joint Program in Ocean Engineering/Applied Ocean Physics and Engineering

September 1, 2006

Certified by

Michael S. Triantafyllou, Professor of Mechanical Engineering Director, Center for Ocean Engineering Thesis Supervisor

Certified by

Franz S. Hover, Principal Research Engineer Thesis Supervisor

Accepted by

Henrik Schmidt, Professor of Mechanical Engineering Chairman, Joint Committee for Applied Ocean Science and Engineering

Accepted by

Lallit Anand, Professor of Mechanical Engineering Chairman, Graduate Committee 


\title{
Emulating the Fast-Start Swimming Performance of the Chain Pickerel (Esox niger) Using a Mechanical Fish Design
}

\author{
By \\ Matthew Nicholas Watts

\begin{abstract}
Submitted on September 1, 2006 in Partial Fulfillment of the
Requirements for the Degree of Master of Science in

Oceanographic Engineering

at the Massachusetts Institute of Technology

and the Woods Hole Oceanographic Institution
\end{abstract}

\section{ABSTRACT}

Mean maximum start-up accelerations and velocities achieved by the fast-start specialist, northern pike, are reported at $120 \mathrm{~ms}^{-2}$ and $4 \mathrm{~ms}^{-1}$, respectively (Harper and Blake, 1990). In this thesis, a simple mechanical system was created to closely mimic the startle response that produces these extreme acceleration events. The system consisted of a thin metal beam covered by a urethane rubber fish body. The mechanical fish was held in curvature by a restraining line and released by a pneumatic cutting mechanism. The potential energy in the beam was transferred into the fluid, thereby accelerating the fish. The fish motion was recorded and the kinematics analyzed while using a number of different tail shapes and materials.

Performance of the mechanical fish was determined by maximum acceleration, peak and averaged maximum velocity, and hydrodynamic efficiency. Maximum start-up acceleration was calculated at $48 \mathrm{~ms}^{-2}$. Peak and averaged maximum velocity was calculated at $0.96 \mathrm{~ms}^{-1}$ and $0.8 \mathrm{~ms}^{-1}$, respectively. The hydrodynamic efficiency of the fish, calculated by the transfer of energy, was $11 \%$. Flow visualization of the mechanical fast-start wake was also analyzed. The visualization uncovered two specific vortex-shedding patterns; a single and a doublevortex pattern are described.

Thesis Supervisor: Michael Triantafyllou Title: Professor of Mechanical Engineering

Thesis Supervisor: Franz Hover Title: Principal Research Engineer 


\section{Acknowledgments}

I would first like to thank my thesis advisors, Michael Triantafyllou and Franz Hover. Their encouragement and insight fostered my successful metamorphous from oceanographer to ocean engineer. They allowed a great deal of freedom and creativity in the design of this project. The approach encouraged a slow and steady confidence in engineering and created a desire to further my educational experience in a number of areas.

Without the permissive time away from sea duty by the US Navy, and funding from the Oceanographer of the Navy, I would never have had this great educational opportunity. The Joint Program between MIT and WHOI is a wonderful educational experience that I will never forget. I am indebted to the naval service for this life-changing experience.

I would especially like to thank Mark Bellanger, manager of the Egerton Center Student Shop. I entered this program without ever using machining equipment or designing any mechanical device. His guidance in the machine shop was paramount in my successful creation of mechanical fish prototypes and my final product. His expertise in CAM and willingness to spend hours over my shoulder allowed me to create a finished product I am quite proud of. I cannot thank him enough for the lessons learned in machining and comfort with design. Frederick Cote was also helpful until his retirement.

I would like to thank Karl Mcletchie for his help in creating the fish curves used for my mechanical fish body. Thank you to Jim Bales of the Edgerton Center for providing the high-speed video recorder and operating lesson. Thank you to Ken Stone of the Hobby Shop for helping me with all my woodwork requirements and teaching me to use the waterjet cutter. Thank you to Karsten Hartel of the Harvard Museum of Comparative Zoology for providing the chain pickerel specimen. Thank you to Pablo Valdivia for the idea to use machinable wax to create my own fish mold. Thank you to Jacob Weisberg who assisted me in carrying out the majority of experiments while I was limited by physical injury.

Members of the Ocean Engineering Towing Tank were always helpful for bouncing ideas and helping me with tools and materials I never imagined using before. Special thanks to current and former MIT graduate students Stephen Licht, Pradya Prempraneerach, David Farrell, Evan Lee, Vicente Fernandez, Matthew Unger, Benjamin Connell, Matthew Greytak, Alexander Bahr, Matthew Walter, David Beal, Dennis Evangelista, Jason Dahl, Benjamin Jones, and Erik Anderson.

My wife, Dawn Watts, has always been a great support system throughout our lives together. The last two years in the Joint Program have been no different. I especially thank her for all her encouragement. 


\section{Contents}

1 Introduction $\quad 13$

$1.1 \quad$ Fast-Start Maneuvers 13

1.2 Body Form Affects Maximum Performance 14

$\begin{array}{lll}1.3 & \text { Thesis Goals } & 14\end{array}$

2 Fish Swimming $\quad 16$

2.1 Fish Swimming Categories 16

2.2 Fish Locomotion Theory 17

2.2.1 Lighthill's Large Amplitude Theory 17

2.2.2 Hydrofoil Theory 18

$\begin{array}{lll}2.3 & \text { Steady Swimming } & 19\end{array}$

2.4 Unsteady Swimming 19

2.5 Fast Starts 20

2.5.1 C-Shape Fast-Start 20

$\begin{array}{lll}2.5 .2 & \text { S-Shape Fast-Start } & 21\end{array}$

$3 \quad$ Fish Form Selection $\quad 22$

3.1 Species Selection 22

3.2 Reported Pike Performance 22

3.2.1 Specialists Uncovered 24

3.3 The Chain Pickerel Model 24

3.3.1 The Body and Head Molds 25

3.3.2 Pike Propulsive Fins 26

4 Design of a C-Shape Mechanical Fast-Start Fish 29

4.1 The Mechanical System 29

4.1.1 Deflected Beam Used For Potential Energy Source 29

4.1.2 Harnessing the Energy of the Deflected Beam 30 
4.1.3 Mechanical Fish Actuation System 30

4.2 Estimating the Mechanical Fish Response 31

4.2.1 Estimating Maximum Velocity 32

4.2.1.1 Energy Conservation 32

4.2.1.2 Hydrodynamic Efficiency 32

4.2.1.3 Elastic Potential Energy 32

4.2.1.4 Kinetic Energy 34

4.2.2 Frequency Response 34

4.2.2.1 $1^{\text {st }}$ Mode Natural Beam Frequency 35

4.2.2.2 Strouhal Number 36

4.2.2.3 Fast-Start Strouhal Number 36

4.2.3 Optimal Radius of Curvature 37

4.2.4 Predictive Use of Equations 38

4.2.4.1 Altering Curvature 38

4.2.4.2 Optimum Prediction 39

4.3 Materials Selected 40

4.3.1 Beam Material $\quad 40$

4.3.2 Mounting Bracket Material 41

4.3.3 Bracket Cover Material 41

4.3.4 Body/Head Mold Material 42

4.3.5 Fish Body Material 42

4.3.6 Tail Fin Mold Material $\quad 42$

$\begin{array}{lll}\text { 4.3.7 Tail Fin Material } & 43\end{array}$

$5 \quad$ Fish Testing $\quad 44$

$5.1 \quad$ Experimental Procedure $\quad 44$

5.2 Data Collection 45

5.2.1 Standard-Speed Imaging 46

5.2.2 High-Speed Imaging 46

$\begin{array}{lll}5.3 & \text { Kinematics Evaluation } & 46\end{array}$ 
$6 \quad$ Testing Results and Discussion $\quad 48$

6.1 Kinematics 48

6.2 Radius of Curvature Tests 49

6.3 Standard-Speed Cinematography Performance Tests 50

6.4 High-Speed Cinematography Performance Tests 52

6.4.1 Cumulative Distance 53

6.4.2 Transverse and Forward Velocities 54

6.4.3 Velocity Smoothing 57

6.4.4 Acceleration 59

6.4.5 Hydrodynamic Efficiency 61

6.4.6 Vortex Wake Patterns 62

6.4.6.1 Double Vortex Pattern 62

6.4.6.2 Single Vortex Pattern 66

$\begin{array}{lll}7 & \text { Future Work and Conclusion } & \mathbf{7 0}\end{array}$

$\begin{array}{lll}7.1 & \text { Future Work } & 70\end{array}$

7.1.1 Including the Preparatory Stroke 70

7.1.2 Fin Form and Position 71

7.1.3 Neutral and Un-Tethered Mechanical Fish 71

7.1.4 Use of Accelerometers 72

$\begin{array}{lll}7.5 & \text { Conclusion } & 72\end{array}$

$\begin{array}{ll}\text { Bibliography } & 74\end{array}$ 


\section{List of Figures}

2.1 Horizontal cut through the wake of a swimming fish

3.1 Photograph of juvenile chain pickerel specimen 25

3.2 Body curves fit to the chain pickerel specimen 26

$\begin{array}{lll}3.3 & \text { Tail fin with a NACA } 0012 \text { profile } & 27\end{array}$

3.4 Tail fin cut to a chain pickerel propulsive fin surface area 28

4.1 Images of bracket, air cylinder cutter, PLC, and solenoid air valves 31

4.2 Effect of beam curvature on forward velocity and energy in the beam 39

4.3 Effect of beam curvature on Strouhal $\tau \quad 39$

4.4 Spring steel cut and shaped for use as the beam material 41

4.5 Mounting bracket and pneumatic air-cylinder $\quad 41$

4.6 Image of the completed fish 42

5.1 Wooden used to place the mechanical fish in curvature 45

6.1 Body traces and COM positions of real and mechanical fish 49

6.2 COM time trace representative of all $200 \mathrm{~Hz}$ tests 52

6.3 COM forward distance covered over time, $200 \mathrm{~Hz}$ testing 53

6.4 Transverse and forward velocity 50-tail 55

6.5 Transverse and forward velocity 60/80-tail 55

6.6 Raw forward velocities of the three high-speed tail tests covering $155 \mathrm{~ms} \quad 56$

$\begin{array}{lll}6.7 & 50 \text {-tail smoothed velocity } & 57\end{array}$

$6.8 \quad 60$-tail smoothed velocity $\quad 58$

$6.960 / 80$-tail smoothed velocity $\quad 58$

6.10 Maximum acceleration using equation for projectile motion 60

6.11 Maximum acceleration using the smoothed velocity derivatives 61

$6.1260 / 80$-tail displaying the double vortex wake pattern 63 
6.1360 -tail displaying the double vortex wake pattern 65

6.14 60-tail displaying the single vortex wake pattern 67 


\section{List of Tables}

3.1 Summary of Esox sp. fast-start performance found in the literature 23

4.1 Constant values used for equations and performance prediction 38

4.2 4.2 Estimated equation values using the design constants from table $4.1 \quad 40$

6.1 Maximum performance derived from the time-distance data at $30 \mathrm{~Hz}$

6.2 Comparison of multiple tail tests at $30 \mathrm{~Hz}$, including deviation 51

6.3 Cumulative forward distance traveled of $200 \mathrm{~Hz}$ tests 54

6.4 Maximum performance parameters from $200 \mathrm{~Hz}$ tests 61 


\section{Chapter 1}

\section{Introduction}

The diversity of fish and marine mammal locomotion has long captured the imagination and research of marine scientists and engineers. Marine biomimetic research is inspired by the ease of fish propulsion when compared with the sluggish movements of most manmade propulsors and propellers.

\subsection{Fast-Start Maneuvers}

The unsteady nature of marine environments and evolutionary pressures found in different aquatic areas has produced certain fish that specialize in unsteady swimming. The accelerations produced by these specialist fish can far outperform manmade aquatic vehicles. For example, Harper and Blake (1990) reported northern pike peak instantaneous accelerations of $250 \mathrm{~ms}^{-2}$. The most extreme acceleration rates occur when fish are escaping life-threatening situations. The maneuver employed by fish in this situation is termed a startle response fast-start.

Fast-starts are quick bursts of energy from fish in a resting or near resting position that achieve high accelerations (Frith and Blake, 1995). Many fish use the startle response faststart to avoid predation. This fast-start produces the greatest accelerations ever recorded of fish. Fish that do not use startle fast-starts rely on other methods, such as camouflage or spines, to avoid predation (Blake, 2004). There are also fish that use fast-starts for prey capture. The accelerations achieved in prey capture have lower peak values, but some 
advantages do exist with this more controlled maneuver. Both responses are well studied with importance to scientists and engineers in fields ranging from evolution to mechanics.

\subsection{Body Form Affects Maximum Performance}

The early studies of fast-starts focused on the kinematics of fish locomotion and reported maximal acceleration values between $40-50 \mathrm{~ms}^{-2}$, regardless of species (Harper and Blake, 1990). The values of cumulative distance, velocity, and acceleration were routinely calculated using distance-time data collected from sequential cinematography images. It has since been proven (Harper and Blake, 1989) that the film methods used in these early studies significantly underestimated peak acceleration rates.

Conflicting with the previous results stating the opposite, Harper and Blake (1990) proved that fish body form is related to fast-start performance. The study used subcutaneously positioned accelerometers and high-speed film analysis of pike and trout to uncover the errors of previous studies. They reported a large disparity in performance between the specialist (pike) and generalist (trout). Overall mean maximum acceleration was $120 \mathrm{~ms}^{-2}$ for the pike and $60 \mathrm{~ms}^{-2}$ for the trout.

\subsection{Thesis Goals}

This thesis is a study to emulate the fast-start performance of fish. A simple-mechanical system was created to closely emulate the startle response of the most successfully studied fast-start specialist species, pike.

The thesis has three goals, to:

- Design a mechanical fish capable of emulating the propulsive stage of the startle fast-start

- Measure the distance traveled, duration, velocity, and acceleration of the mechanical fish

- Measure the efficiency of the mechanical fish 
Evaluating the mechanical system created for this thesis provides a basis for further work on manmade fast-start accelerators. Applying the ability to quickly accelerate to ocean vehicles could improve turning ability, start-up/braking performance, and maneuvering in turbulent environments. With the ever-increasing need for quality scientific and strategic sampling in hard to reach regions, increased unsteady performance of vehicles is an approaching requirement. 


\section{Chapter 2}

\section{Fish Swimming}

\subsection{Fish Swimming Categories}

Fish swim by positively interacting with the water around them. They create hydrodynamic forces by generating reactive forces through body undulations and by shedding momentum into the fluid from their tails and fins (Wakeling, 2001). Fish have evolved numerous methods of creating these forces over millions of years. Sorting the myriad of fish adaptations in the aquatic environment is no simple task. The approach taken by Webb (1984) was to characterize fish locomotion according to the functional propulsive mechanisms they employ.

Webb’s (1984) four swimming categories are body and caudal fin (BCF) periodic, BCF transient, median and paired fin (MPF), and occasional or non-swimmers. This classification incorporates the predatory characteristics corresponding to the main propulsive mechanism. For example, tuna use the BCF periodic mechanism in a swimming style suited for long distance high-speed fish. In order to overcome a wide food distribution in the open ocean, these fish must travel long distances in space and time. BCF transient, like pike, are periodic swimmers that also have a specialization in the area of fast starting or maneuvering. Their prey should be considered more abundant spatially, but more evasive than the previous case. The MPF and occasional swimmers are typically found in more complex environments. Their food is less evasive and the more dynamic environment has a profound impact on body form. 


\subsection{Fish Locomotion Theory}

Locomotion in an aquatic environment is far different from what is experienced on land. It is Newton's laws of motion; the third law in particular, along with Archimedes' law and Bernoulli's equation that govern the forces exchanged in the life aquatic. For fish locomotion, they must accelerate water away from their bodies to achieve the oppositedirected propulsion.

The absence of a solid surface to provide a reactive force for efficient locomotion requires the creation of a virtual surface in the fluid. Imparting momentum into the fluid by controlled movements of the body and fins create such a 'surface'. Two theories on fish swimming are briefly explained to describe fish locomotion.

\subsubsection{Lighthill's Large-Amplitude Theory}

Lighthill's (1971) large-amplitude elongated body theory for fish swimming describes lateral undulations of a fish body to achieve forward propulsion (Videler, 1993). The fish produces symmetric undulations that impart both lateral and thrust forces into the water. The amplitude of the undulating fish increases towards the tail fin, affecting more of the surrounding water. The lateral forces cancel, due to symmetry, while forward thrust remains. The largeramplitude lateral motion of the tail region imparts an increasing change in momentum to the surrounding fluid. This creates a momentum jet directed opposite the swimming direction.

Simply stated, water forced away from the fish provides the reactive force that propels it. The greater the amount of water affected by the undulation, the stronger the propulsive jet. Therefore, fish with large posterior surface areas create large added mass effects associated with the undulation. This increases the amount of momentum transferred into the fluid to provide thrust. 


\subsubsection{Hydrofoil Vortex Theory}

The vortex theory of fish swimming describes tail-induced forces on a fluid in much the same manner as aerofoil theory (Videler, 1993). Like an aerofoil, the tail fin has a rounded leading edge and a sharp trailing edge. During steady swimming, an angle of attack between the oncoming flow and the tail fin is created when the fin is moved laterally through the water. This angled attack causes stagnation of the flow and an increased pressure on one side of the tail. Increased flow speed and lower pressure on the opposite side of the tail causes rotation of the fluid about the sharp trailing edge to equalize the pressure differential. The rotation needed to hold the stagnation point at the sharp trailing edge is described by the Kutta condition.

The rotation imparts vorticity into fluid along the entire length and span of the tail fin. The rotating fluid is termed the bound vortex. Circulation in the vortex is clockwise when the tail moves to the right and anti-clockwise when the tail moves left (figure 2.1). The vortex gathers strength throughout the tail beat, and terminates the instant the tail stops or changes direction. The terminated vortex is shed into the fluid to allow the opposite-signed vortex to begin. In steady swimming, the cyclic process of creating and shedding these vortices into the wake provides continued forward thrust.

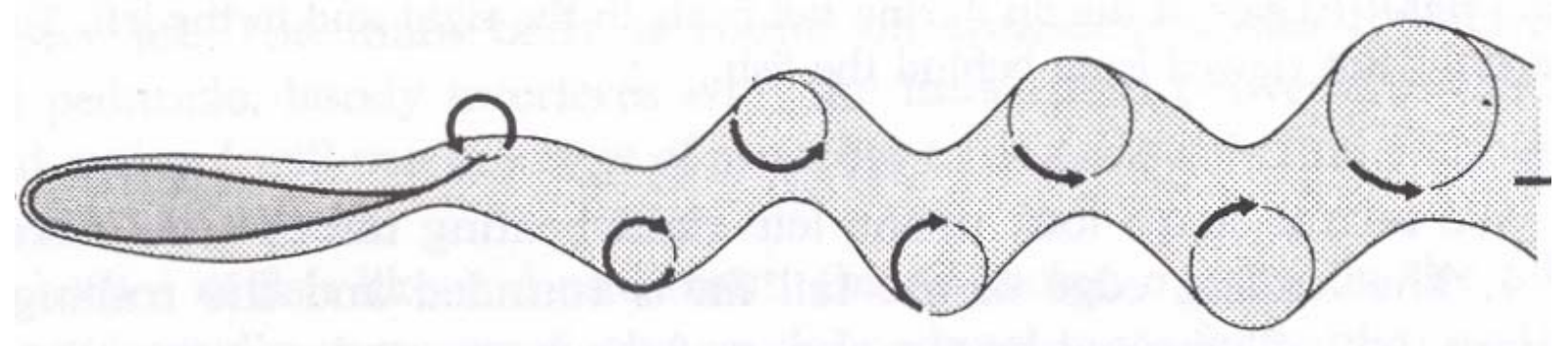

Figure 2.1 A horizontal cut through the wake of a swimming fish. Vortices are shed from the tail, imparting momentum into the surrounding fluid. The vortices alternate direction as the tail flips from side to side. (From Videler, 1993)

Figure 2.1 displays a horizontal cut through the wake of a swimming fish. The shed vortices are shown alternating direction and decreasing in strength as they spread through the fluid. Following the circulation arrows, an undulating propulsive jet could be traced 
oscillating through the vortices. Lighthill (1969) theorized the existence of a propulsive jet providing a continuous thrust to the swimming fish. His inviscid theory further described the strength of momentum in the jet as equal and opposite in direction to the momentum of the fish (Videler, 1993).

\subsection{Steady Swimming}

The swimming modes of fish are broadly characterized in this thesis as steady and unsteady swimming.

Steady swimming refers to the absence of acceleration. Examples of steady swimming are observed in fish that are patrolling or transiting from point to point, maintaining a steady velocity and direction. Under such circumstances, steady swimming specialists, like yellowfin tuna, are reported to swim at speeds of at least 40 knots (Triantafyllou and Triantafyllou, 1995). The propulsive mechanism they utilize is body and caudal fin periodic.

The fish swimming theories (2.2) described above are presented as steady swimming cases. In addition to the swimming theories, both steady and unsteady swimmers utilize vorticity control to increase efficiency. Vorticity control allows fish to maximize their interactions with the surrounding fluid. Vortex manipulation allows fish to constructively use their proximal environment, even their own wake, to increase performance and produce large hydrodynamic forces (Triantafyllou and Triantafyllou, 1995). This is particularly the case in unsteady swimming, described below.

\subsection{Unsteady Swimming}

Fish are rarely in an environment that allows continuous velocities and a steady supply of non-evasive food. It is for this reason that fish have evolved over millions of years to optimally manipulate their dense-fluid environment. The unsteady swimming that is required of fish includes fast-starts, braking, and rapid turning. The hydrodynamic performance of these behaviors is governed by the same principles of reactive and circulatory (shed vortices) 
forces as explained for steady swimming (Wakeling, 2001). However, they also require an ability to actively manipulate the vortices created by their own bodies to achieve peak performance.

Blake (2004) noted that the kinematics and mechanics are essentially the same for Ctype fast-starts and for turns. The primary difference is the existence of oncoming flow in the turning case. This similarity suggests an improved understanding of any one of the unsteady maneuvers could offer significant advance in all of them. Further explanation of fast-start kinematics and hydrodynamics are discussed in greater depth below, assuming the principles could be translated to all unsteady behavior.

\subsection{Fast-Starts}

Fast-starts are characterized by their purpose and body shape kinematics. The purpose of the start can either be a predatory or escape response. Weihs described each fast-start maneuver broken into three distinct stages (Ahlborn and others, 1991, Blake, 2004). The first is the preparatory stage. It consists of a quick contraction of the fish and lends the kinematics description, either $\mathrm{C}$ or $\mathrm{S}$. The propulsive stage, stage two, is the aggressive uncoiling of the fish to produce the desired locomotion. The final stage is a variable phase that may include subsequent propulsive strokes or simply coasting (Ahlborn et al., 1997).

\subsubsection{C-Shape Fast-Start}

The escape response is initiated when a fish is startled or attacked. To ensure survival, the fish requires its greatest possible velocity in the shortest time. The fish activates the Mauthner neuron that simultaneously activates all of the muscles on one side of the body. This sends a wave of bending traveling along the fish towards the tail (Wakeling, 2001). The kinematics description of this maneuver is a C-shape.

The C-start preparatory stroke creates large-unbalanced forces which cause the center of mass to rotate and the fish to accelerate (Blake, 2004, Wakeling, 2001). The propulsive stroke, a single tail flap, creates a force directed backward to produce the greatest forward acceleration. Straightening the tail causes continued rotation of mass while the fish escapes in 
a direction away from the initiating stimulus. Domenici and Blake (1991) noted that maximal acceleration is critical during the escape response, while direction is not.

\subsubsection{S-Shape Fast-Start}

Some fish employ a fast-start mechanism used for prey capture. It requires the predator to accelerate in a direct path towards its prey. This start can occur from rest or while the fish is steady swimming. The kinematics is described by the S-shape curvature created by the fish body in the preparatory stroke. The forces generated in this feeding preparatory stroke are more balanced along the fish body and eliminate any uncontrolled turning (Blake, 2004). The propulsion phase is a burst in the prey direction, generally consisting of a full-cycle tail beat followed by single tail flaps until prey capture (Harper and Blake, 1991). Following prey capture, the fish enters the coasting phase. Maximum acceleration and velocity are lower than the Mauthner initiated escape fast-start (Hale, 2002), but feeding fast-starts maximize performance while maintaining directional stability throughout the maneuver (Harper and Blake, 1991). 


\section{Chapter 3}

\section{Fish Form Selection}

\subsection{Species Selection}

Fast-start performance is dependent on body form (Harper and Blake, 1990). Specialists of fast-starts have a body form that is coincident with hydrodynamic theory. Theory states that good performance is enhanced by lateral profiles designed to maximize the amount of water accelerated by body motions (Blake, 2004, Frith and Blake, 1995). Long and slender bodies provide large amplitudes and high angles of attack at the thrust producing areas of the body and tail fin (Frith and Blake, 1995). By this standard, pike are better designed for fast-start maneuvers than a swimming generalist, i.e. trout. Therefore, the body form of the mechanical fish in this thesis was modeled from a pike species.

\subsection{Reported Pike Performance}

Table 3.1 displays maximum performance data of Esox species from the literature (Harper and Blake, 1990, Dominici and Blake, 1997). The fast-start type studied in each paper is presented in the table as either an escape response (ER) or a feeding strike (FS). The table is a compilation of performance studies using a number of different collection methods. The predominant method was video imaging (frame rate provided in $\mathrm{Hz}$ ). The authors used kinematics time-distance data to calculate performance parameters. The other method used was subcutaneously placed accelerometers (Acc) at the fish center of mass. The accelerometers have a $125 \mathrm{kHz}$ frequency response, a very high sampling rate. 
Maximum velocity is given as specific velocity (fish lengths per second) and in $\mathrm{ms}^{-1}$. Fish velocity and distance traveled are both size dependent, so specific velocity is often used to compare performance between different sized specimens. However, fast-start acceleration is not size dependent, and is given in $\mathrm{ms}^{-2}$ (Dominici and Blake, 1997). The duration of stages one and two, the preparatory and propulsive strokes, are combined and provided in milliseconds. The table clearly displays earlier studies using kinematics reported maximum accelerations much lower than those from the more recent studies implementing accelerometry.

Table 3.1 Summary of Esox sp. fast-start performance found in the literature.

\begin{tabular}{c|c|c|c|c|c|c|c} 
Authors & $\begin{array}{c}\text { Common } \\
\text { Name }\end{array}$ & $\begin{array}{c}\text { Fast- } \\
\text { Start } \\
\text { Type }\end{array}$ & $\begin{array}{c}\text { Method } \\
(\mathrm{Hz})\end{array}$ & $\begin{array}{c}\text { Maximum } \\
\text { Acceleration } \\
\left(\mathrm{ms}^{-2}\right)\end{array}$ & $\begin{array}{c}\text { Maximum } \\
\text { Velocity } \\
\left(\mathrm{Ls}^{-1}, \mathrm{~ms}^{-1}\right)\end{array}$ & $\begin{array}{c}\text { Duration } \\
(\mathrm{ms})\end{array}$ & $\begin{array}{c}\text { Body } \\
\text { Length } \\
(\mathrm{m})\end{array}$ \\
\hline Weihs (1973) & Pike & ER & 40 & 50 & - & - & - \\
\hline Webb (1978) & $\begin{array}{c}\text { Tiger } \\
\text { musky }\end{array}$ & ER & 250 & 39.5 & $7.2,1.6$ & 115 & 0.217 \\
\hline $\begin{array}{c}\text { Rand and } \\
\text { Lauder } \\
(1981)\end{array}$ & $\begin{array}{c}\text { Chain } \\
\text { pickerel }\end{array}$ & FS & 200 & 21.1 (mean) & $9.0,2.5$ & 92 & 0.273 \\
\hline $\begin{array}{c}\text { Webb (1986) } \\
\text { Tiger } \\
\text { musky }\end{array}$ & ER & 60 & 15 (mean) & $21,1.4$ & - & 0.065 \\
\hline $\begin{array}{c}\text { Harper and } \\
\text { Blake (1991) }\end{array}$ & $\begin{array}{c}\text { Northern } \\
\text { pike }\end{array}$ & ER & Acc & 120.2 (mean) & $10.5,3.97$ & 108 & 0.378 \\
\hline $\begin{array}{c}\text { Harper and } \\
\text { Blake (1991) }\end{array}$ & $\begin{array}{c}\text { Northern } \\
\text { pike }\end{array}$ & FS & Acc & $\begin{array}{c}95.9 \\
\text { (mean) }\end{array}$ & $8.2,3.1$ & 133 & 0.378 \\
\hline $\begin{array}{c}\text { Frith and } \\
\text { Blake (1991) }\end{array}$ & $\begin{array}{c}\text { Northern } \\
\text { pike }\end{array}$ & ER & 250 & 151.3 (mean) & $8.7,3.5$ & 129 & 0.400 \\
\hline
\end{tabular}

As shown in table 3.1, published maximum accelerations using film measurements for pike escapes were estimated at $40-50 \mathrm{~ms}^{-2}$ before the 1990's. The same approximate maximum values were also reported in studies for the generalist swimmer, trout, during the same periods. The results presented from a 1990 study by Harper and Blake (1991) proved that the fast-start specialist pike greatly outperforms the generalist trout. It also proved that hydrodynamic theory holds true, and body form is a significant factor in fast-start performance. 


\subsubsection{Specialists Uncovered}

The Harper and Blake (1991) study of escape responses compared subcutaneously positioned accelerometers in rainbow trout, Salmo gairdnerei, and northern pike, Esox lucius, with accelerations derived by visual double integration. The comparison came following a report by the same authors (1989) outlining the errors associated with high-speed cinematography for acceleration measurements. Using single-axis accelerometers, mean maximum acceleration rates of $120.2 \mathrm{~ms}^{-2}$ and $59.7 \mathrm{~ms}^{-2}$ for pike and trout were presented, respectively. These values were compiled from 25 pike and 30 trout fast-starts.

Focusing on the Esox species, the 25 pike escape fast-starts were divided into three categories by different kinematics variations and performance. The variation in escape behavior showed that pike employs escapes that are often less than maximal. This may correspond to the degree of danger the pike interprets, and the performance needs it will require to avoid the offending stimulus. A ‘true maximal' escape category was created which included seven of the 25 analyzed fast-starts. Of those seven, a single-event maximum acceleration rate of $244.9 \mathrm{~ms}^{-2}$ was achieved. The mean maximum velocity and acceleration in this extreme category was $4.70 \mathrm{~ms}^{-1}$ (+/-0.52) and 157.8 (+/-37.3), respectively.

Further mention of maximum performance will refer to the lumped values presented in table 3.1 values, not the subdivision of extreme fast-starts listed in the previous paragraph. That data was presented to explain what some of the recent literature has cited as the maximal performance of northern pike, 25 g's.

\subsection{The Chain Pickerel Model}

The mechanical fish was designed in three parts. The head was designed to allow easy access to the pneumatic cutting system and to easily change body materials without recasting the head. The body was accurately modeled after a chain pickerel, and the mold was designed to accept different material thickness if needed. Two tail shapes were created and tested with differing surface areas; the tail fins were created for easy attachment with little alteration to the body. 


\subsubsection{The Body and Head Molds}

The body form used to model the mechanical fish was the chain pickerel, Esox niger. A deadjuvenile specimen was obtained from Harvard's Museum of Comparative Zoology

(Cambridge, MA) and photographed (figure 3.1). The fish photographs were fit with polynomial curves of the body outlines, excluding the fins. For symmetry, the profiles from the top and bottom curves were averaged (figure 3.2). A 3-Dimensional Computer Aided Design (CAD) program, SolidWorks, was used to import the profiles and loft an ellipse along the fish curves, creating the 3-D model.

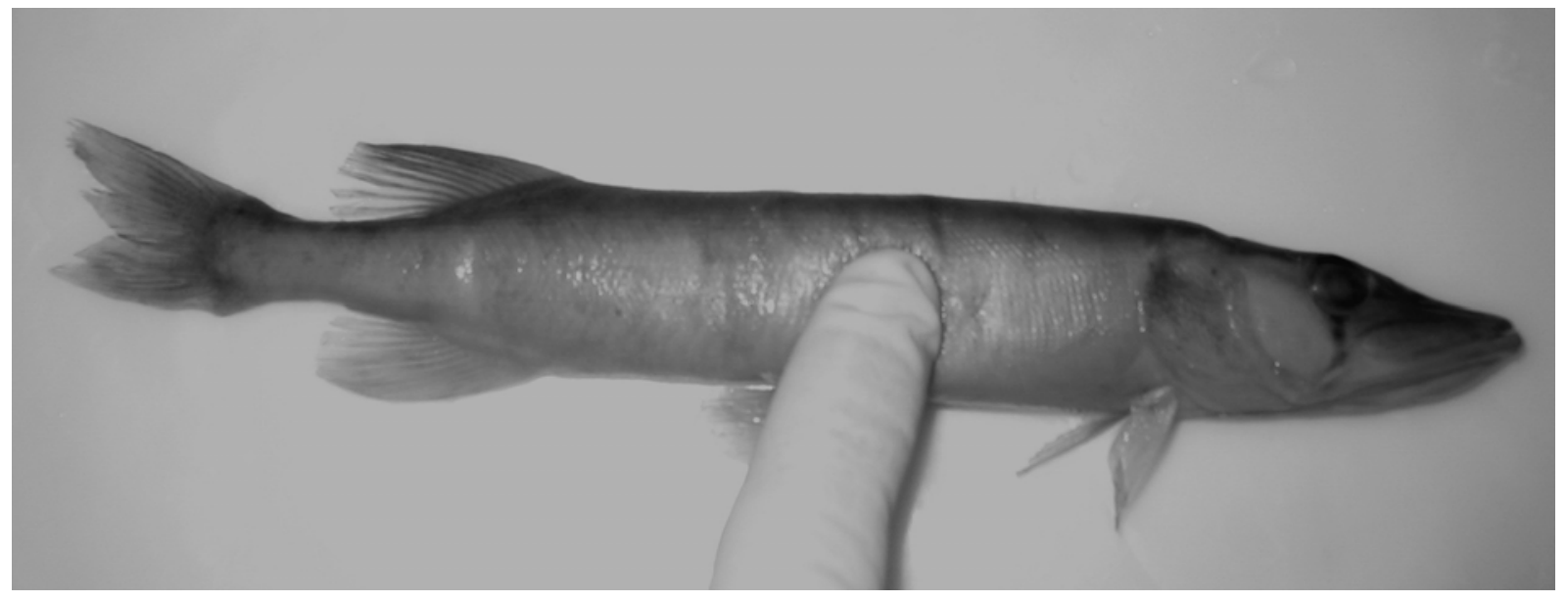

Figure 3.1 Photograph of juvenile chain pickerel specimen obtained from the Harvard Museum of Comparative Zoology.

The 3-D chain pickerel model was used to create a fish mold for multiple rubber castings. A solid mold was fit around the fish model in the CAD program and exported into Computer Aided Machining (CAM) software, Mastercam. Following tool paths, feed-rates, and bit selection, the fish mold was machined. A Bridgeport EZ-Track Computer Numerical Controller (CNC) was used to machine the wax in two parts. Separation between the head, body, and tail of the mechanical fish was a result of design and function. 


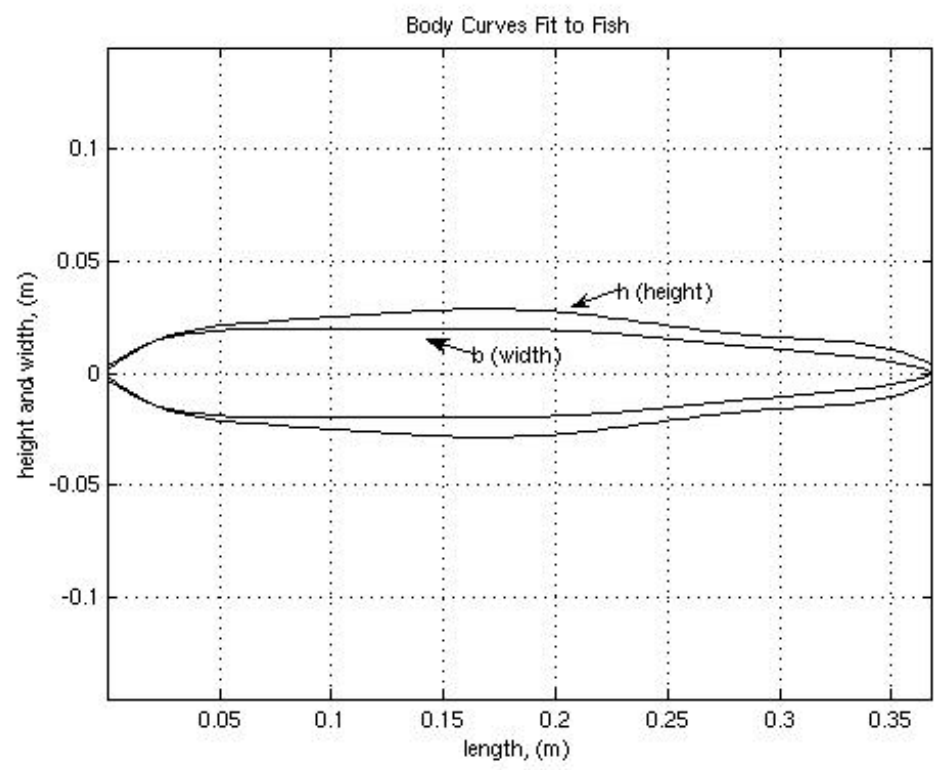

Figure 3.2 Body curves fit to the chain pickerel specimen.

The 3-D chain pickerel model was used to create a fish mold for multiple rubber castings. A solid mold was fit around the fish model in the CAD program and exported into Computer Aided Machining (CAM) software, Mastercam. Following tool paths, feed-rates, and bit selection, the fish mold was machined. A Bridgeport EZ-Track Computer Numerical Controller (CNC) was used to machine the wax in two parts. Separation between the head, body, and tail of the mechanical fish was a result of design and function.

Using separated components for the head, body, and tail allowed for controlled alterations to the system. The head mold separation allowed access to the actuation mechanism and attachment to different beam materials possible. The separated body mold ensured the ability to exchange beam material or thickness without complete re-casting of the fish. Tail separation from the body also allowed the exchange in material and shape while keeping other factors consistent.

\subsubsection{Pike Propulsive Fins}

The pike species incorporate three prominent posterior-positioned fins used for thrust production: the anal, dorsal and caudal fins (Frith and Blake, 1995). The large surface area 
provided by these lifting surfaces allows the pike to reach the impressive start-up accelerations and velocities described above [3.2].

Incorporating three propulsive fins with differing stiffness and position on the mechanical fish was abandoned for a single lifting surface. An assumption was made that the surface area of a single fin could reasonably approximate the effect of the same area split between three individual surfaces.

The single tail fin used for testing the mechanical fish is a NACA 0012 profile (figure 3.3). The tail fin mold was originally designed by Dr. David Beal and created for comparison between 2-dimensional and 3-dimensional fin shape. Dr. Beal's mold was slightly altered to incorporate a small mounting plate to connect the tail with the beam for this thesis. The maximum span and chord measurements are $19.7 \mathrm{~cm}$ and $9.5 \mathrm{~cm}$, respectively. The estimated surface area for a fin of these dimensions is $187 \mathrm{~cm}^{2}$.

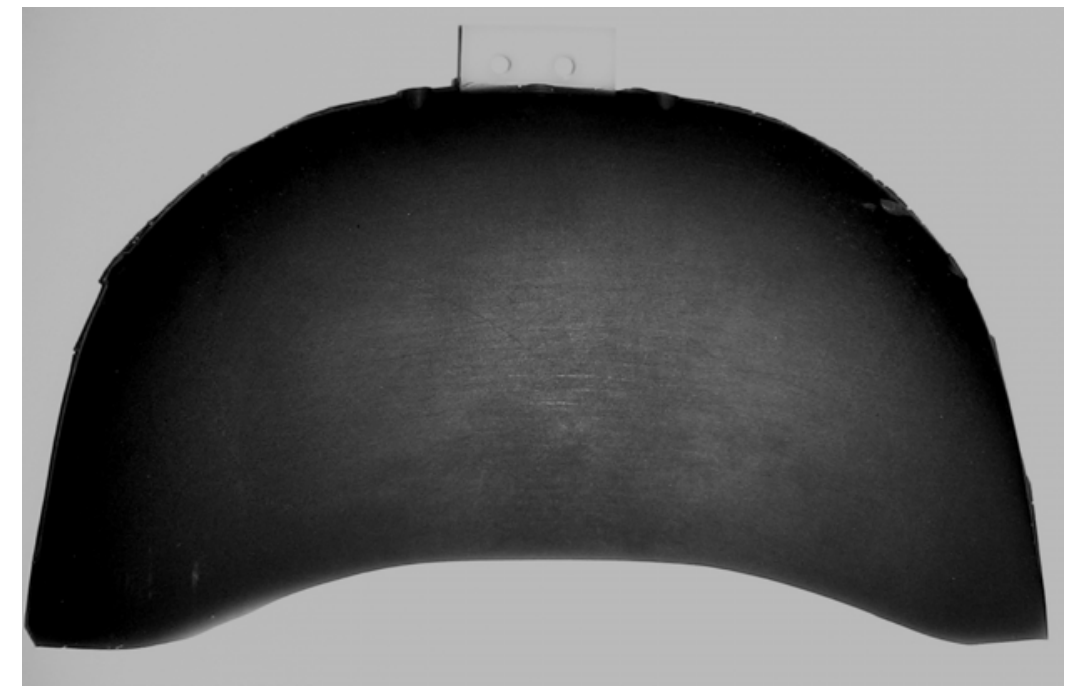

Figure 3.3 Tail fin with a NACA 0012 profile.

The propulsive fin surface area of a chain pickerel of comparable size to the mechanical fish is half the size of Beal's fin. The approximate surface area of a comparable pike is $76 \mathrm{~cm}^{2}$. This value was found estimating the surface area of the juvenile pike specimen and assuming linear growth. Limiting the tail fin to a more correct size required symmetrically trimming the top and bottom of the fin (figure 3.4). Edge effects associated with this procedure are not evaluated. 


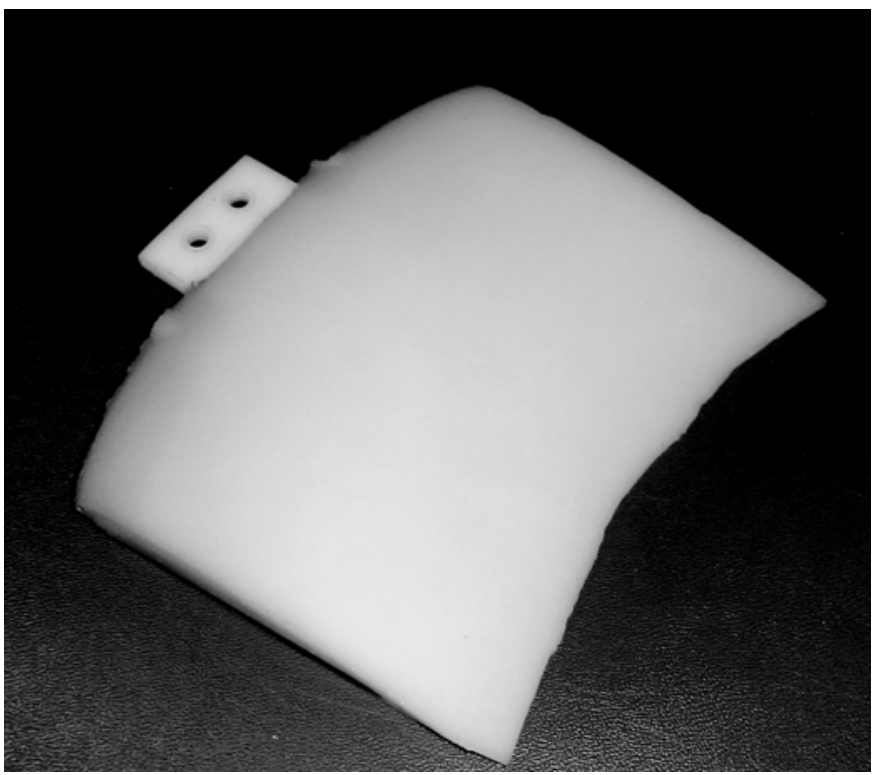

Figure 3.4 Tail fin cut to a size representative of the surface area for the chain pickerel propulsive fins. 


\section{Chapter 4}

\section{Design of a C-Shape Mechanical Fast-Start Fish}

The design goal was to create a simple mechanical fish capable of achieving fast-start performance characteristics similar to those of real fish. However, fish are complicated subjects to mimic; this forced a simplification of the complex kinematics and focused the effort on the dominant thrust-producing action. In the C-shape fast-start, the thrust phase occurs directly following the fish's coiling into the C-shape. Therefore, the mechanical fish was not designed to produce a preparatory phase and coil on its own.

\subsection{The Mechanical System}

\subsubsection{Deflected Beam Used For Potential Energy Source}

Looking exclusively at the C-shape fast-start propulsive phase of fish, there is a quick and efficient transfer of energy from the fish to the water. The muscles of a real fish actively produce this energy transfer. To emulate this effect mechanically, a large amount of potential energy must be harnessed, maintained, and quickly transferred into the water producing forward thrust. The fast-start kinematics of a real fish is similar in basic form to a deflected beam with free ends that elastically deformed and released. This similarity, and the relative simplicity of calculating the beam response, made its application for propulsion a feasible option. 


\subsubsection{Harnessing the Energy of the Deflected Beam}

Design of the mechanical fish in this thesis was developed from the 'beam' up. A beam, in this case a thin sheet of material, placed in curvature is the only source of mechanical potential energy for the system. This restriction of design required a method to hold and release the energy, on command, to accomplish the goal of forward propulsion. To hold the beam in curvature, steel anchors are used to securely attach low-stretch fluorocarbon fishing line (Seaguar Invisible Leader Material, 0.026 in. diameter) from head to tail. The use of fishing line for holding the mechanical fish coincided with the actuation system employed for release, or firing.

\subsubsection{Mechanical Fish Actuation System}

The actuation system of the mechanical fish is a pneumatic cutter attached to the beam by an aluminum-mounting bracket (figure 4.1 (a)). A pneumatic cutting mechanism was one of the least problematic of the proposed actuation options. The action of cutting the restraining line immediately removes the deflecting force without any residual opposing forces. In preliminary designs, multiple cutters were employed onboard the fish in an attempt to better match real fish kinematics. Up to three cutters were used at 1/10-second intervals progressing from the tail tip through the body. The combination of limited release time-intervals and interruption of the dominant beam motion focused the project to a single release system.

The cutting mechanism, displayed in figure 4.1 (b), is composed of an air-cylinder (Bimba original line 5/16-inch bore) fitted with a trimmed chiseling blade (X-acto \#17). The cylinder is powered by an off-fish air compressor (Husky 4 gallon tank mounted portable) and connected using flexible air tubing (Freelin-Wade 5/32 in. outer diameter, 3/32 in. inner diameter). A Programmable Logic Controller (PLC) (Crouzet Millinium II ${ }^{+}$SA12) connected to a solenoid air-valve (MAC 34C-AAA-GDCA-1KV) provided precise air control (figures 4.1 (c and d).

The air cylinder is made from stainless steel and does not require any waterproofing for our testing depth. Its cutting ability during our tests was also unaffected by flooding prior 
to firing. The greatest disadvantage incurred with this actuation method was the requirement to restring the fish between firings.

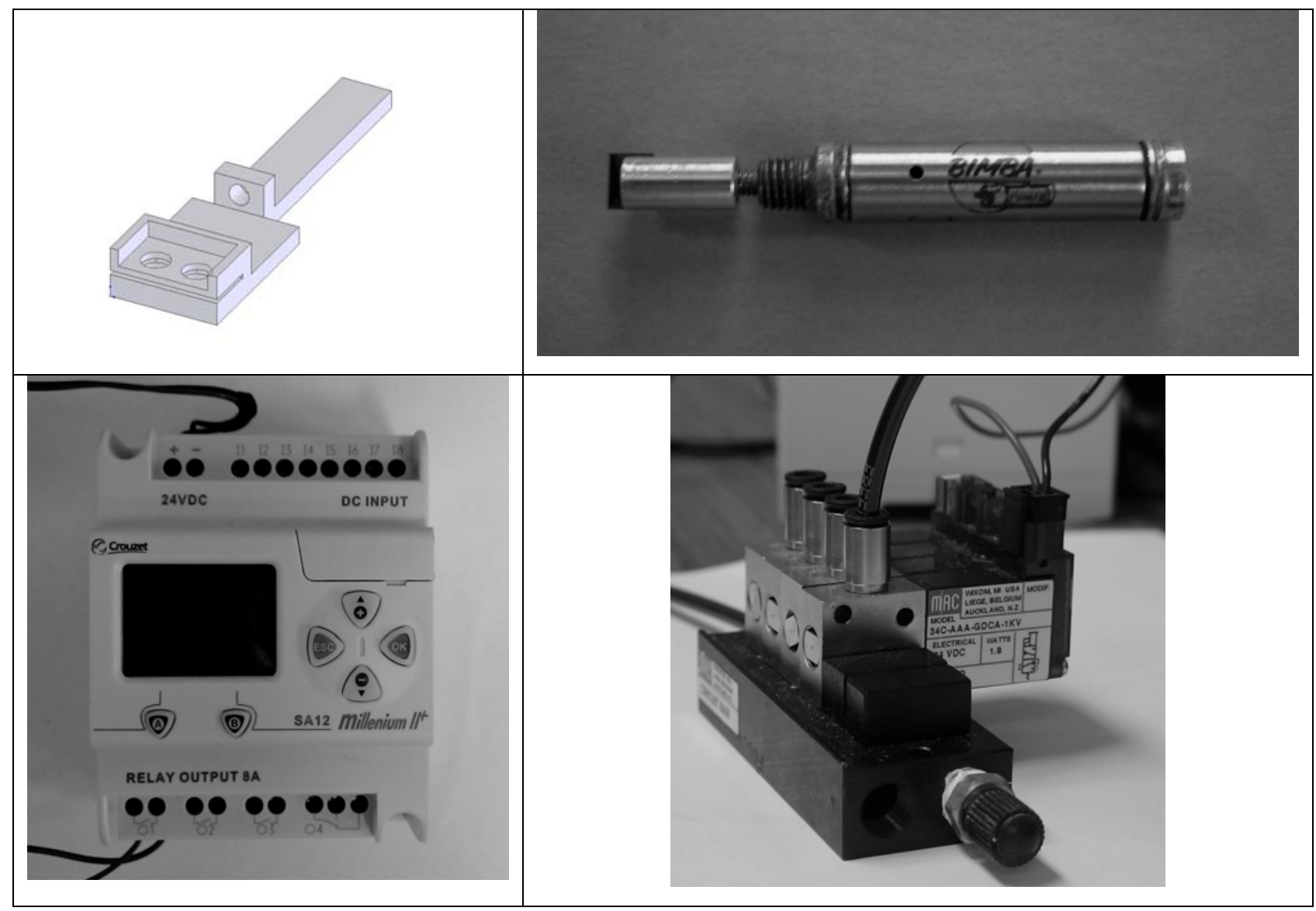

Figure 4.1 Moving clockwise from the top left: (a) Image of the aluminum-mounting bracket used to attach the pneumatic cutter with beam. (b) Pneumatic cutting mechanism, Bimba air cylinder with Xacto blade mounted to the rod. (c) Image of the solenoid air valves used to precisely power the air cylinder. (d) Image of the Crouzet PLC used to operate the solenoid air valves.

\subsection{Estimating the Mechanical Fish Response}

With a design for the mechanical fast-start fish in place, some analysis was required to estimate fish performance before fabrication. Estimating the response of different materials provided an educated approach to choosing materials. The particular parameters of interest were the maximum velocity, the time for one efficient tail beat, and the amount of curvature required to produce them. 


\subsubsection{Estimating Maximum Velocity}

\subsubsection{Energy Conservation}

The fish design uses a curved (deflected) beam to store mechanical potential energy $\left(E_{p}\right)$ in the system. When the beam is released from curvature it will quickly transfer its energy into the surrounding fluid. A portion of the energy released from the beam will become useful kinetic energy and provide the fish with forward propulsion. The rest of the energy is 'lost' into the fluid and the structure as heat. Regardless of the energy distribution, the energy conservation law must always be satisfied. An equation for kinetic energy $\left(E_{k}\right)$ is:

$$
E_{k}=\eta \cdot E_{p}
$$

A hydrodynamic efficiency constant, $\eta$, is included in the equation above to account for the energy 'loses' into the fluid. Hydrodynamic efficiencies of pike fast-starts are reported between 0.16 and 0.39 using a ratio of useful power to total power (Frith and Blake, 1995). Expecting a lower end value during the mechanical testing, an efficiency constant of 0.2 was used in the analysis.

\subsubsection{Hydrodynamic Efficiency}

The highest efficiency values of fish locomotion are realized in steady swimming; they range between 0.7 to 0.9 (Frith and Blake, 1995). The fish velocities under which these efficiencies are reported range from 4-8 $\mathrm{BLs}^{-1}$ (BL, body lengths). Decreases in efficiency are observed as the propulsive mechanism becomes more unsteady. For example, McCutcheon reports burst-and-coast swimming efficiency ranging from 0.18 to 0.7 (Frith and Blake, 95). A faststart is a violent-unsteady propulsive mechanism, causing efficiency values to decrease accordingly.

\subsubsection{Elastic Potential Energy}

Elastic potential energy in a one-dimensional stretching material is given by: 


$$
E_{p}=\int \frac{E \cdot A_{0} \cdot \Delta l}{l_{0}} d l
$$

The elastic modulus $(E)$ is the stiffness of a material and defines the amount of force required to bend. The cross sectional area $\left(A_{0}\right)$ is the inertia $(I)$ of the material shape. The material length $\left(l_{0}\right)$ is stretched by some amount $(\Delta l)$ to produce the mechanical potential.

The mechanical potential energy stored in a deflected beam is dependent on the material properties, dimensions, and the degree of deflection. Applying equation 4.2 to a beam with constant curvature produces the following equation of potential energy in polar coordinates.

$$
\frac{d \theta}{d s}=\text { constant }=\frac{1}{\mathrm{R}}
$$

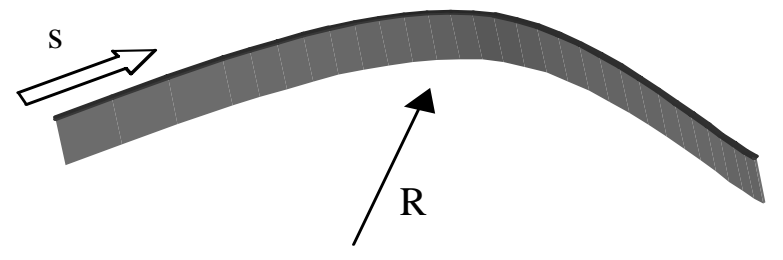

$$
\begin{gathered}
E_{p}=\int_{0}^{l_{\text {beam }}} E I \cdot\left(\frac{d \theta}{d s}\right) d s=\frac{1}{2} \cdot E I \cdot\left(\frac{1}{R}\right)^{2} \cdot l_{\text {beam }} \\
I=\frac{b \cdot h^{3}}{12}
\end{gathered}
$$

The beam dimensions are width $(b)$, thickness $(h)$, and length $\left(l_{\text {beam }}\right)$. The inertia of a flat beam is found using the cross-sectional area of a rectangle. The degree of deflection is defined using the radius of curvature $(R)$ of the initial deflection.

The assumption of constant curvature is applied to the system for ease of calculation. The beam, in actuality, does not configure itself to a perfect radius. The ends of the beam, where the restraining line is anchored, will tend to straighten and therefore reduce the curvature. The potential energy calculation described here will tend to over estimate the energy stored in the beam when deflected. 


\subsubsection{Kinetic Energy}

The energy that provides the mechanical fish with forward propulsion is considered useful energy to the system. The kinetic energy is the energy required to do work, given as:

$$
\begin{gathered}
E_{k}=\frac{1}{2} \cdot m_{\text {forward }} \cdot U^{2} \\
m_{\text {forward }}=m_{\text {fish }}+0.2 \cdot m_{\text {fish }} \\
m_{\text {fish }}=\pi \cdot \frac{b_{\text {fish }}^{2}}{8} \cdot \rho_{\text {rubber }} \cdot l_{\text {fish }}
\end{gathered}
$$

The kinetic energy calculation incorporates the properties of the completed mechanical fish, not just beam specific properties. It requires the mass of the fish in the forward direction ( $m_{\text {forward }}$ ) and the maximum velocity $(U)$. The $m_{\text {forward }}$ equation (4.7) is the combination of the total mass of the fish $\left(m_{\text {fish }}\right)$ and the added mass effect caused by accelerating the fluid around the fish. Webb (1982) (Frith and Blake, 1995) experimentally derived the added mass associated with forward fish acceleration as $20 \%$ of the total mass. The $m_{\text {fish }}$ is approximated by half the mass of a cylinder with diameter $b_{f i s h}$, total fish length $l_{\text {fish }}$, and a rubber body with density $\rho_{\text {rubber }}$.

Solving for the estimated maximum fish velocity is possible by substituting the expanded energy equations (4.4 and 4.6) into the conservation equation (4.1):

$$
\begin{gathered}
\frac{1}{2} \cdot m_{\text {forward }} \cdot U^{2}=\frac{1}{2} \cdot E I \cdot \frac{1}{R^{2}} \cdot l_{\text {beam }} \cdot \eta \\
U=\sqrt{\frac{\eta \cdot E I \cdot l_{\text {beam }}}{R^{2} \cdot m_{\text {forward }}}}
\end{gathered}
$$

\subsubsection{Frequency Response}

Optimizing the natural beam response is crucial to emulate fast-start fish performance. A combination of the beam material and beam dimensions coincides with a frequency that should produce the greatest velocity. Estimating the correct combination requires evaluating the beam natural frequency and matching it with the average fast-start frequency of real fish. 


\subsubsection{1 $1^{\text {st }}$ Mode Natural Beam Frequency}

The natural response frequency of the design beam is estimated using the dynamic beam equation with free-end boundary conditions. The equation describes the vertical deflection $z(x, t)$ as a function of space and time:

$$
m \ddot{\mathscr{X}}=-E I z^{\prime \prime \prime \prime}
$$

Dots and primes correspond with partial time derivatives and spatial derivatives, respectively.

The boundary conditions that describe a beam with free ends are zero moment and zero shear at $\mathrm{x}=0$ and $\mathrm{x}=l_{\text {beam }}$. Applying the boundary conditions and solving for the spatial non-trivial solutions produce the characteristic equation:

$$
\cos (\beta) \cosh (\beta)=1
$$

Solving the above equation describes the infinite roots used to find the harmonic frequencies:

$$
\beta_{n}=\frac{(2 n+1) \cdot \pi}{2}
$$

The $n^{\text {th }}$ natural frequency of vibration associated with the $n^{\text {th }}$ mode shape of a free-free beam is given by:

$$
\omega_{n}=\frac{\left(\beta_{n}\right)^{2}}{L^{2}} \cdot \sqrt{\frac{E I}{M}}
$$

The first mode of vibration found through beam mechanics $\left(\omega_{1 \_ \text {beam }}\right)$ is of particular interest; it approximates the same shape as the beam in constant curvature:

$$
\begin{gathered}
\omega_{1_{-} \text {beam }}=\frac{\left(\frac{3}{2} \pi\right)^{2}}{l_{\text {beam }}^{2}} \cdot \sqrt{\frac{E I}{M}} \\
M=m_{\text {beam }}+m_{a_{-} \text {beam }}=b \cdot h \cdot \rho_{\text {beam }}+b^{2} \cdot \frac{\pi}{4} \cdot \rho_{\text {water }}
\end{gathered}
$$

Because the beam is acting in water, the mass $\left(m_{\text {beam }}\right)$ and added mass $\left(m_{a \_b e a m}\right)$ of the beam is included. For $m_{a \_b o d y}$, the fish shape is assumed to be a cylinder with diameter equal to the beam width, $b$. The values of mass and added mass are given per unit length as $M$ (total mass per unit length). 


\subsubsection{Strouhal Number}

The non-dimensional Srouhal number is used to describe and compare oscillating flow mechanisms.

$$
S t=\frac{f \cdot A}{U}
$$

The Strouhal number (St) is defined by frequency $f$, amplitude $A$, and forward velocity $U$. The Strouhal number is used in this thesis as a frequency parameter to help with analysis. In order to predict an optimum radius of curvature for the deflected beam, real fast-start frequencies are included in the prediction by using equation 4.17.

\subsubsection{Fast-Start Strouhal Number}

The Strouhal number of real fast-start fish $\left(S t_{f s}\right)$ was calculated from data provided by Schriefer and Hale (2004) on escape responses in northern pike. The average length of the examined fish was $23.7 \mathrm{~cm}$. The mean linear velocity for the escapes was $1.75 \mathrm{~ms}^{-1}$ with a propulsive stage duration of $29.5 \mathrm{~ms}$. The frequency of the average startle response calculated from the duration is $33.9 \mathrm{~Hz}$. The amplitude of the tail excursion was approximated using the kinematics figure 2 from Schriefer and Hale (2004). The approximate value is 0.4 of the fish length.

Applying the above values provides an approximate fast-start frequency Strouhal number:

$$
S t_{f s}=\frac{33.9 \cdot(0.4 \cdot 0.237)}{1.75}=1.84
$$

Implementing the non-dimensional Strouhal number for the fast-start fish produces a frequency equation for the mechanical fish.

$$
S t \Rightarrow 1.84=\frac{f \cdot A}{U} \Rightarrow f=\frac{1.84 \cdot U}{0.4 \cdot l_{\text {fish }}} \Rightarrow f=4.6 \cdot \frac{U}{l_{\text {fish }}}
$$

The duration $(\tau)$ of the propulsive tail oscillation and the circular frequency $(\omega)$ are given by:

$$
\begin{gathered}
\tau=\frac{1}{f} \\
\omega=2 \pi \cdot f
\end{gathered}
$$


Using the assumed optimal escape response Strouhal number of 1.84, a second frequency equation is provided $\left(\omega_{1 \_s t}\right)$. This frequency equation incorporates the velocity equation $U$ and the total length of the designed fish.

$$
\omega_{1 \_S t}=\frac{9.2 \cdot \pi}{l_{\text {fish }}} \sqrt{\frac{\eta \cdot E I \cdot l_{\text {beam }}}{R^{2} \cdot m_{\text {forward }}}}
$$

\subsubsection{Optimal Radius of Curvature}

The energy that fuels the mechanical fish is introduced by deflecting the stiff beam. The greater the deflection, the more energy that is imparted to the system. For the fish to optimally perform, it must efficiently transfer the potential energy stored in the beam into kinetic energy. The correct amount of curvature will allow the fish to perform maximally. However, there is a limit to the energy the fish will efficiently transfer, and a point where increasing deflection further will not improve performance.

Equating the fundamental duration $\tau_{\text {beam }}$ of the deflected beam with the calculated Strouhal duration $\tau_{S t}$ identified for optimal fast-starts provides a theoretical optimal curvature, $R_{\text {opt }}$. Barring physical limitations of the system, components, or the user, the fast-start fish will be optimally tuned with the fluid using this specified amount of deflection.

$$
\begin{gathered}
\tau_{\text {beam }}=\tau_{\text {St }} \\
\frac{2 \cdot \pi}{\frac{2.25 \cdot \pi^{2}}{l_{\text {beam }}^{2}} \cdot \sqrt{\frac{E I}{M}}}=\frac{l_{\text {fish }}}{4.6 \cdot \sqrt{\frac{\eta \cdot E I \cdot l_{\text {beam }}}{R^{2} \cdot m_{\text {forward }}}}} \\
R_{\text {opt }}=\frac{l_{\text {fish }}}{4.09 \cdot l_{\text {beam }}^{2}} \frac{1}{\sqrt{\frac{M \cdot m_{\text {forward }}}{\eta \cdot l_{\text {beam }}}}}
\end{gathered}
$$




\subsubsection{Predictive Use of Equations}

Predicting values of the above governing equations required setting constants for the design fish. Table 4.1 displays the constant values used for performance predictions.

Table 4.1 Constant values used for equations and performance prediction.

\begin{tabular}{|c|c|l|}
\hline Notation & Value & \multicolumn{1}{c|}{ Description } \\
\hline$E$ & $200 \mathrm{GPa}$ & Modulus of Elasticity \\
\hline$I$ & $5.63 \times 10^{-12} \mathrm{~m}^{-4}$ & Beam inertia \\
\hline$b$ & $0.0330 \mathrm{~m}$ & Beam width \\
\hline$h$ & $0.00127 \mathrm{~m}$ & Beam thickness \\
\hline$b_{\text {fish }}$ & $0.0635 \mathrm{~m}$ & Fish width \\
\hline$l_{\text {beam }}$ & $0.368 \mathrm{~m}$ & Beam length \\
\hline$l_{\text {fish }}$ & $0.508 \mathrm{~m}$ & Fish length \\
\hline$\rho_{\text {beam }}$ & $7850 \mathrm{kgm}^{-3}$ & Beam density \\
\hline$\rho_{\text {water }}$ & $1000 \mathrm{kgm}^{-3}$ & Water density \\
\hline$\rho_{\text {rubber }}$ & $1000 \mathrm{kgm}^{-3}$ & Rubber density \\
\hline$m_{\text {fish }}$ & $0.804 \mathrm{~kg}$ & Fish mass \\
\hline$m_{\text {forward }}$ & $0.965 \mathrm{~kg}$ & Fish forward mass \\
\hline$\eta$ & 0.2 & Hydrodynamic efficiency \\
\hline$M$ & $1.184 \mathrm{kgm}^{-1}$ & $\begin{array}{l}\text { (Mass + added mass) per } \\
\text { unit length }\end{array}$ \\
\hline
\end{tabular}

\subsubsection{Altering Curvature}

The equations for potential energy, maximum velocity, and the calculated Strouhal frequency and duration are all dependent on curvature. A changing radius of curvature was evaluated using a constant arc length (s) while changing the angle $\theta$ :

$$
\begin{aligned}
& R=\frac{s}{\theta} \\
& s=l_{\text {beam }}
\end{aligned}
$$

The angle was first evaluated from 0 to $\pi$, from no curvature to a complete C-shape.

However, the angles of interest that produced velocities between 0.5 and $2 \mathrm{~ms}^{-1}$ are $\pi / 6$ to $7 \pi / 9\left(30^{\circ}-140^{\circ}\right)$. These angles correspond to radii of curvature from $0.6-0.15 \mathrm{~m}$. Figure 4.2 displays the estimated velocity and potential energy required as a function of the radius of curvature. Figure 4.3 displays the duration of the propulsive stroke calculated by the Strouhal ratio. 


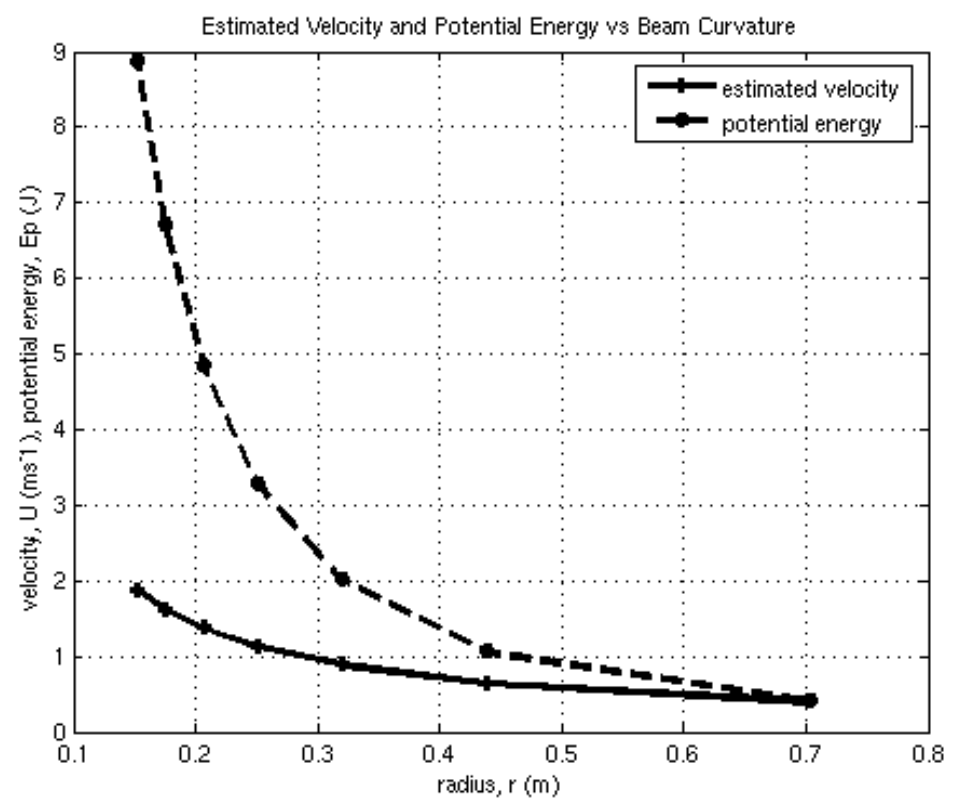

Figure 4.2 Effect of beam curvature on forward velocity and energy in the beam.

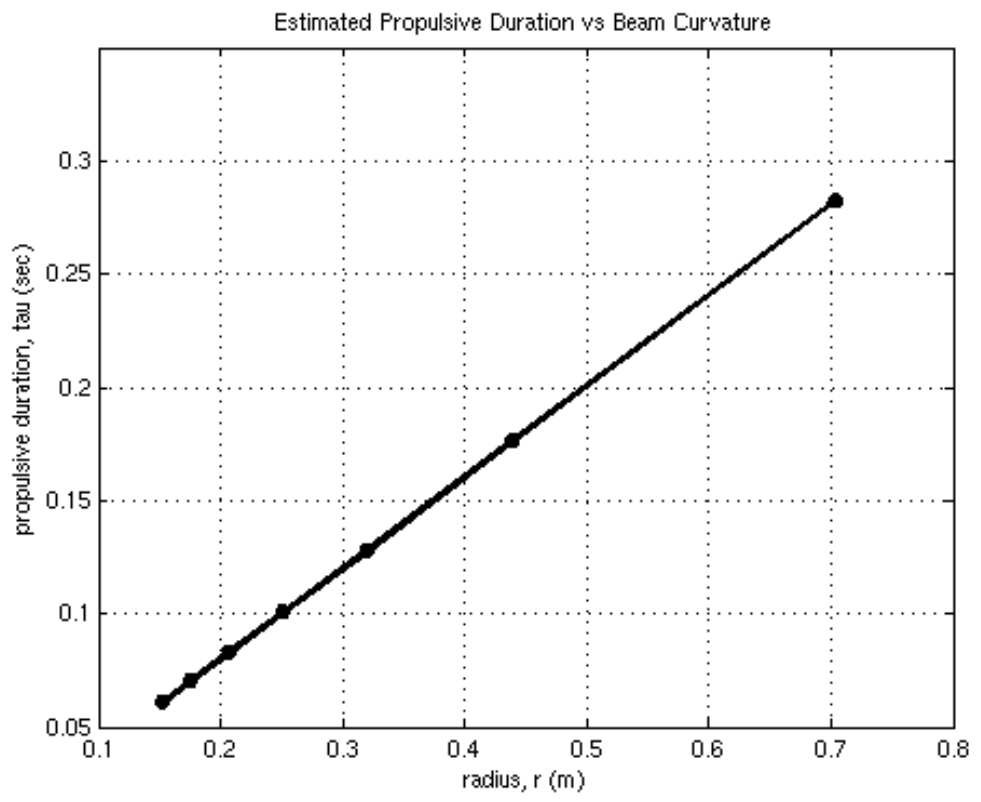

Figure 4.3 Effect of beam curvature on Strouhal $\tau$.

\subsubsection{Optimum Prediction}

The optimum radius of curvature was calculated at $0.233 \mathrm{~m}$ using the $R_{\text {opt }}$ equation described above. This value corresponds to approximately $90^{\circ}$ of curvature. This radius was used to 
calculate estimated optimal values displayed in table 4.2 from the equations above. Also displayed in this table are beam mechanics values independent of curvature.

Table 4.2 Estimated equation values using the design constants from table 4.1.

\begin{tabular}{|c|c|l|}
\hline Notation & Value & \multicolumn{1}{|c|}{ Description } \\
\hline$R_{\text {opt }}$ & $0.233 \mathrm{~m}$ & Radius of curvature \\
\hline$S t_{\text {real }}$ & 1.84 & Strouhal number, fast-start \\
\hline$E p$ & $3.82 \mathrm{~J}$ & Potential energy \\
\hline$E k$ & $0.76 \mathrm{~J}$ & Kinetic energy \\
\hline$U$ & $1.26 \mathrm{~m} / \mathrm{s}$ & Forward velocity \\
\hline$f_{\text {St }}$ & $12.88 \mathrm{~Hz}$ & Frequency, Strouhal \\
\hline$f_{\text {beam }}$ & $25.45 \mathrm{~Hz}$ & Frequency, mechanics \\
\hline$\tau_{\text {St }}$ & $0.078 \mathrm{sec}$ & Duration, Strouhal \\
\hline$\tau_{\text {beam }}$ & $0.039 \mathrm{sec}$ & Duration, mechanics \\
\hline$\tau_{\text {fish }}$ & $0.088 \mathrm{sec}$ & Duration, propulsive estimate \\
\hline
\end{tabular}

\subsection{Materials Selection}

Material selection was generally based on price, availability, and ease of machining. However, some governing equation analysis was required for proper selection of the beam material and dimensions. Using common materials insured the availability of proper machining within the MIT community. A goal of this thesis was to accomplish all machining by the author. Access to lathes, mills and water jet cutters was available through the Edgerton Student Machine Shop and the MIT Hobby Shop.

\subsubsection{Beam Material}

Blue-finished 1095 spring steel (McMaster-Carr) was used for the beam material. It is a material with good bounce-back properties and wear-resistance, able to withstand the repetitive bending required for this application. The hardened-steel does require special attention when machining. An OMAX water jet cutting table was used to cut the spring steel to the required size and shape.

The raw material thickness was 0.05 in, with 2 in. width. The steel was cut to 14.75 in. by 1.3 in. for use as the mechanical fish beam. Eight holes were also cut into the beam. Four of the holes were used for mounting the head bracket and tail fin to the beam. Three 
holes were used to ensure a solid physical connection of the body material to the beam. The last hole is a slot for connecting the restraining line with the beam (figure 4.4).

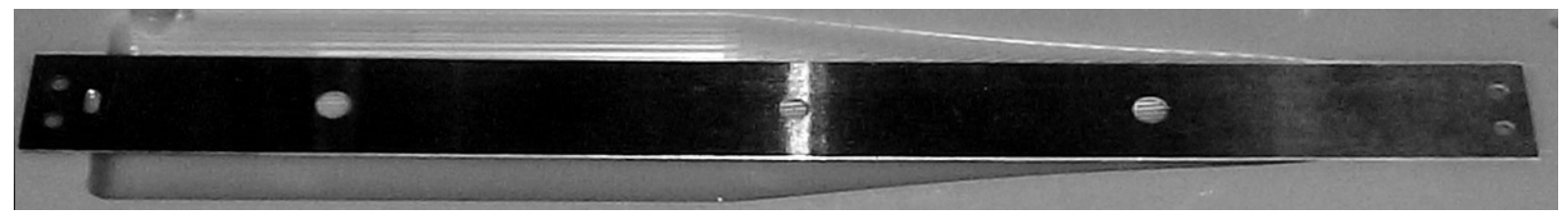

Figure 4.4 Spring steel cut and shaped for use as the beam material of the fast-start fish.

\subsubsection{Mounting Bracket Material}

The mounting bracket used to attach the pneumatic cutter with the beam was machined from a multipurpose aluminum alloy (alloy 6061). It was chosen for its strength, good corrosion resistance and fair machinability. The bracket was designed for a single air-cylinder and a single restraining line connection. The bracket was slotted for an on-centerline fit with the beam. The bracket was tapped for a solid connection with the cylinder and the beam-securing screws (figure 4.5).

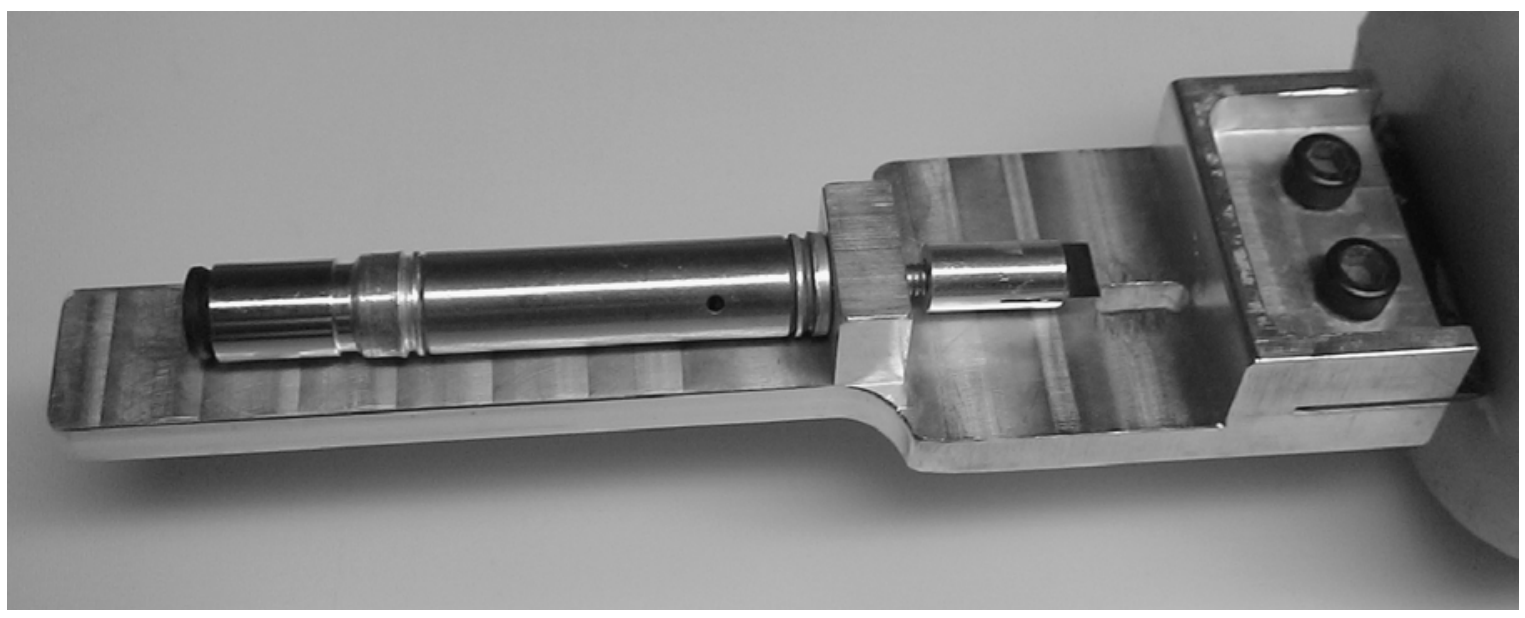

Figure 4.5 Mounting bracket used to connect the pneumatic air-cylinder to the fish body.

\subsubsection{Bracket Cover Material}

Urethane foam ('butterboard', McMaster-Carr) was used to seal the bracket from the pouring compound of the head mold. The urethane cover acts like a glove over the head bracket. It is 
then cast in rubber, using the head mold, to match the body material and profile. The butterboard also provided added buoyancy without fluid absorption.

\subsubsection{Body/Head Mold Material}

Machinable wax (Freeman Manufacturing and Supply) was used for the fish molds. The wax is a hard-non-porous material that is easy to machine. The molds were machined using a Bridgeport EZ-Track CNC. The smooth-finished surface allowed easy separation between the mold and the cast rubber.

\subsubsection{Fish Body Material}

The fish body-material was selected by its density and hardness properties, often coincident when using Smooth-On ${ }^{\circledR}$ brand urethane rubbers. The VytaFlex ${ }^{\mathrm{TM}}$ series of rubbers are a 1:1 volume mixed low-viscosity rubber. The low viscosity minimizes air-bubble entrapment and the 1:1 mixing ratio simplifies mixing. The greatest flexibility found in this rubber series, VytaFlex 10 (Shore A Hardness 10, density $1000 \mathrm{~kg} / \mathrm{m}^{3}$ ), was used as the body and head rubber. This rubber provides the fish a realistic body without introducing significant energy into the system when bent. A complete fish image is displayed in figure 4.6.

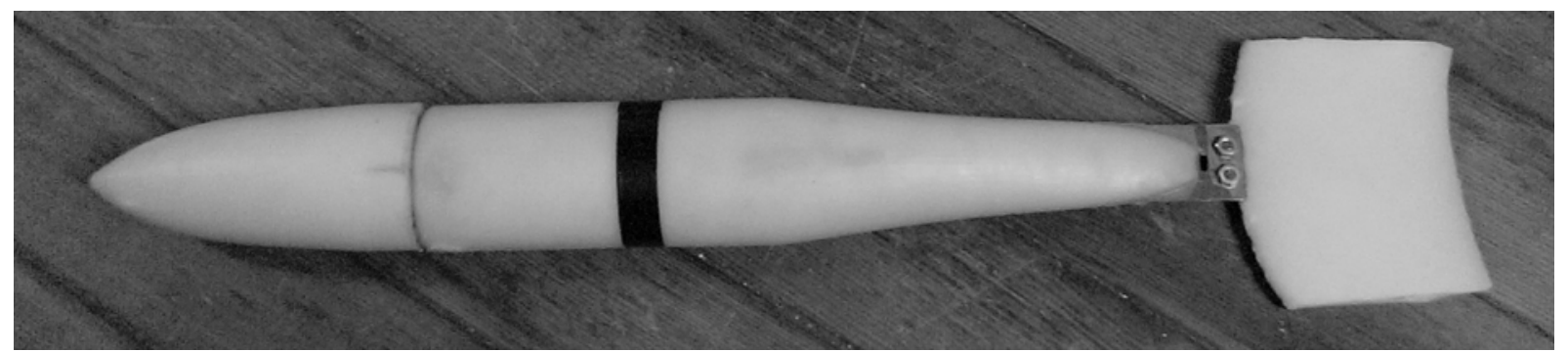

Figure 4.6 Completed mechanical fish.

\subsubsection{Tail Fin Mold Material}

The tail fins used in this thesis were cast using a NACA 0012 profile mold. The shape of the tail is of generic form created by David Beal, a former MIT graduate student. 


\subsubsection{Tail Fin Material}

The tail-fin material was selected to cover a wide variety of possible tail stiffness. They

include Shore A Hardness 50 (VytaFlex 50), 60 (VytaFlex 60), 80 (Smooth-On PMC-780), 94 (Freeman), and Shore D Hardness 70 (Smooth-Cast 310). The first four listed are urethane rubbers and the last is a urethane plastic. The rubber and plastic were cast onto Delrin inserts used to securely attach the metal beam. A full tail and cut tail image are available in figures 3.3 and 3.4. 


\section{Chapter 5}

\section{Fish Testing}

Comparison of the mechanical fish with real fish performance required comparable calculation methods. The distance-time method for calculating distance traveled, velocity, and acceleration was employed with both 30 and 200 frames $\mathrm{s}^{-1}$ cinematography. The efficiency of the mechanical fish was estimated using the kinetic energy of the fish divided by the elastic potential energy in the system.

\subsection{Experimental Procedure}

Testing of the mechanical fish was carried out in a 350-gallon glass tank (94.38 x $29.25 \mathrm{x}$ 29.25 in.) at the MIT towing tank laboratory. Testing required a compressed air supply to operate the actuation mechanism onboard the fish. Imparting the required elastic potential energy into the fish required a preparation jig (figure 5.1). The fish was placed in curvature using the plywood jig and a band clamp moments before attaching to the air system. While attached to the jig, the fishing line was inserted and anchored through the head bracket at one end and the tail region at the other. Holding the fish in curvature for an extended time placed unnecessary stress on the fluorocarbon fishing line, and often resulted in premature firing.

The 'loaded' mechanical fish was taken to the tank and the air tube inserted into the cylinder. The molded head was then placed on the bracket and hung into the tank. The fish was attached to a mounting string approximately $1 \mathrm{~m}$ long. The string held the negatively buoyant fish suspended in the water horizontally. Firing the fish required an impulse from the 
PLC to the air valve, relaying 120 psi of air pressure into the air cylinder, finally cutting the fishing line.

The Mechanical fish testing focused on the effect of different tail materials and shapes. The tail materials ranged from 50 to 94 Shore A hardness for rubber tails, and a plastic tail with 70 Shore D hardness. There was also a combination tail created by casting the 80 A material above the 60 A material. The idea of the combination tail was to stiffen the leading edge of the tail fin with the 80 A while taking advantage of the more elastic properties of the 60 A material. The test sizes were either a full tail or a cut tail. The full tail was oversized while the cut tail was better proportioned to the mechanical fish.

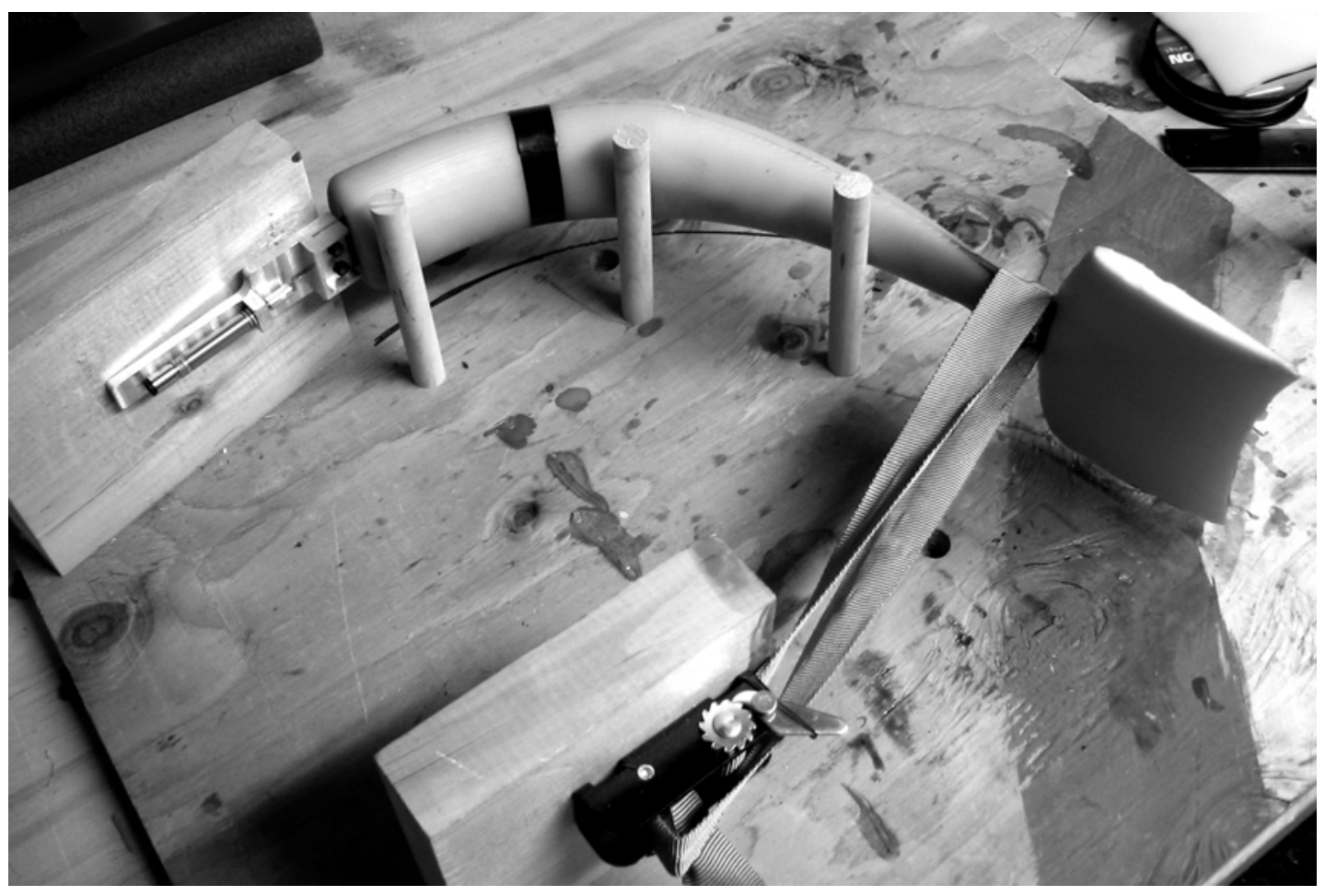

Figure 5.1 Wooden jig and band clamp used to place the mechanical fish in curvature.

\subsection{Data Collection}

Analyzing digital film images of the mechanical fish kinematics was the method used to evaluate performance. Initial data collection was limited to commercial film rates. Two 
standard Sony cameras were used and their data was evaluated. Intrigued by the exceptional performance values reported for the real fish of the species being emulated, the need for a higher film rate was evident. A high-speed digital video recorder was used for the last experiments of this thesis.

All digital video images were imported into a video editing program, iMovie HD or Phantom, and converted to QuickTime format. Evaluating the fish kinematics provided the two primary parameters of interest, the velocity and the acceleration. Dye experiments were also completed and shed wake vortices evaluated.

\subsubsection{Standard-Speed Imaging}

The majority of fast-starts were recorded at 30 frames per second from an observation window (camera one) below the fish and from a camera (camera two) mounted beside the tank. The images from below the fish provided the kinematics of the fast-start maneuver, while the horizontally mounted camera provided a qualitative view of the fast-start. Camera one was a Sony DCR-HC1000 digital video camera recorder. A 0.45x's wide conversion lens, VCL-SW04, was attached for an improved field of view. Camera two was a Sony DCRDVD403 digital video camera recorder.

\subsubsection{High-Speed Imaging}

The fast-start mechanical fish was recorded at 200 frames per second from the observation window located below the testing tank. A Phantom v7 (camera three) digital high-speed video camera was borrowed from the Edgerton Educational Center at MIT. The images were used to precisely evaluate several fast-starts.

\subsection{Kinematics Evaluation}

Placing a transparency over the computer screen while replaying the movie clip provided a straightforward calculation of the fish velocity from the kinematics. First, a scaling value ( $\varsigma$ ) of the real fish $\left(l_{\text {real }}\right)$ to the digital fish image ( $\left.l_{\text {image }}\right)$ was calculated using a scaling standard. 
The standard was a $3 / 4$ inch colored-tape strip that marked the center of mass (COM) position on the fish.

$$
\frac{l_{\text {real }}}{l_{\text {image }}}=\varsigma
$$

Tracing the COM position of the fish over time and properly scaling the distance between images provided an averaged velocity. The distance between COM's in consecutive images is $\vec{d}_{\text {image }}$, and the film rate is the incremental time $(t)$ between images.

$$
\vec{v}=\frac{\vec{d}_{\text {image }} \cdot \varsigma}{t}
$$

Calculating an averaged acceleration from the kinematics required the double integration of distance (equation 5.3) or the equation of projectile motion (5.4). $\vec{a}$ is the acceleration rate, $\vec{v}_{i}$ is the initial velocity, and $\vec{x}_{i}$ is the initial position.

$$
\begin{gathered}
\vec{a}=\frac{\delta \vec{v}}{\delta t} \\
\vec{x}=\frac{1}{2} \vec{a} \cdot t^{2}+\vec{v}_{i} \cdot t+\vec{x}_{i}
\end{gathered}
$$

Efficiency of the mechanical fast-start fish is a comparable performance characteristic of interest. The hydrodynamic efficiency ( $\eta$ ) is a ratio of the kinetic energy of the released fish to the potential energy of the fish in curvature.

$$
\eta=\frac{E_{k}}{E_{p}}
$$




\section{Chapter 6}

\section{Testing Results and Discussion}

Comparison of the mechanical fish with real fish performance required comparable calculation methods. The distance-time method for calculating distance traveled, velocity, and acceleration was employed with both 30 and 200 frames $\mathrm{s}^{-1}$ cinematography. The efficiency of the mechanical fish was estimated using the kinetic energy of the fish divided by the elastic potential energy in the system.

\subsection{Kinematics}

The mechanical fish performance is provided in two categories: beam mechanics and fish locomotion. Using a stiff beam as the energy supply instead of controllable muscles, like fish, causes a significant change in the kinematics. A qualitative display of the center of mass (COM) motion and the whole body kinematics are presented for both real and mechanical fish.

In figure 6.1, the left set of body tracings are from Webb (1975) and the right side are from the fast-start mechanical fish. It is evident from this figure how the fish is able to control the caudal region and to produce a smooth transition from the $\mathrm{C}$ or L-shape position to a straight path of motion. The traces from Webb are separated by $15 \mathrm{~ms}$. On the right, the beam tracings are separated by $33 \mathrm{~ms}$ and it is clearly displayed how difficult matching fish kinematics is without a higher level system. The dots on the tracings correspond with the COM of the fish. The fish COM moves predominantly inline with the fish's intended 
swimming direction, while the mechanical fish has much greater 'slip' motion. The scale bar located below the tracings is $10 \mathrm{~cm}$.

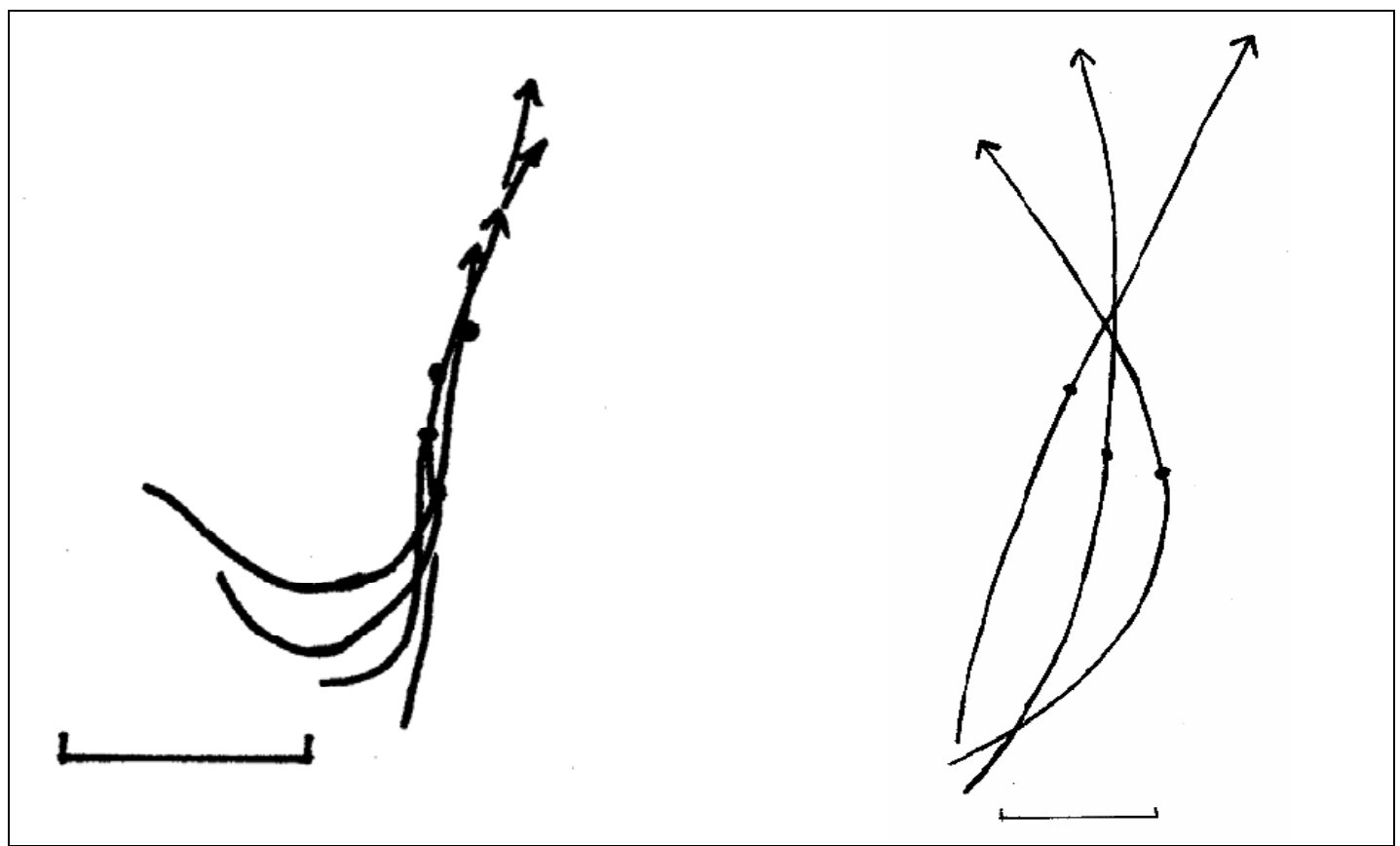

Figure 6.1 Body traces with COM marked with dots. The tracings on the left are of an L-shape faststart by trout without a high acceleration turn, $15 \mathrm{~ms}$ separation. The right tracings are of the mechanical fish based on $30 \mathrm{~Hz}$. Scale bars are $10 \mathrm{~cm}$.

\subsection{Radius of Curvature Tests}

The first sequence of repetitive testing performed with the mechanical fast-start fish was altering the restraining line length. These tests consisted of changing the length of the fluorocarbon fishing line anchored to the fish head and tail, controlling the beam curvature. The fishing line test lengths ranged from $24-34 \mathrm{~cm}$. The results were reviewed from digital video and evaluated qualitatively.

It was found that a physical limit existed for line lengths below $27 \mathrm{~cm}$. The lines were either snapped immediately or soon after attaching to the fish. This limit was most likely caused by insufficient line test strength (50 lb test). Also, the mechanical system itself had areas with higher line strain and thin edges where the line passed through the beam. Nevertheless, the limit occurred beyond the original length of interest that provided $90^{\circ}$ of curvature $(28 \mathrm{~cm})$. 
The bulk of systematic testing of the mechanical fish used $28 \mathrm{~cm}$ restraining line that held the fish along a $23 \mathrm{~cm}$ constant radius curve. All tests reported below were completed using $28 \mathrm{~cm}$ restraining line.

\subsection{Standard-Speed Cinematography Performance Tests}

A combination of six tail materials and two tail shapes were used to calculate fast-start escape performance of the mechanical fish. The majority of tail testing results relied on standardspeed $\left(\sim 30\right.$ frames s$\left.^{-1}\right)$ digital video recorded during the test runs. The velocities and accelerations are derived from the time-distance data as described in the procedure. Table 6.1 displays the maximum velocities and maximum accelerations calculated for each test run.

The numbers and letters in the tail column refer to the size and material hardness scale of each tail. Full (F) tails are oversized, while cut (C) tails more accurately represent the surface area of real pike propulsive fins [3.3.2]. The 60/80 tails were an attempt to stiffen the leading edge of the fin with the 80 A material while allowing keeping the flexibility of the 60 A material at the trailing end [5.1]. All tails have a NACA 0012 hydrofoil profile. Columns for locomotive acceleration and velocity refer to the fish COM motion in the final escape direction. Columns for the beam acceleration and velocity refer to the maximum scalar values of the center of mass, dominated by beam mechanics.

For tail comparison, table 6.2 displays the peak values of acceleration and velocity for each type. Mean values and the standard deviations are given where multiple test runs of the same tail were evaluated.

Comparing the tails in table 6.2, the best overall performers are the 50,60, and combination 60/80. A scientific conclusion on the fast-start performance of the mechanical fish is not possible using consumer speed video data. A closer examination into the kinematics and subsequent calculated performance of the mechanical fish required high-speed video imaging. 
Table 6.1 Maximum performance parameters derived from the time-distance data at $30 \mathrm{~Hz}$.

\begin{tabular}{|c|c|c|c|c|c|}
\hline Run & $\begin{array}{c}\text { Tail } \\
\text { (Hardness } \\
\text { scale) }\end{array}$ & $\begin{array}{c}\text { Locomotive } \\
\text { Acceleration } \\
\left(\mathrm{ms}^{-2}\right)\end{array}$ & $\begin{array}{c}\text { Beam } \\
\text { Acceleration } \\
\left(\mathrm{ms}^{-2}\right)\end{array}$ & $\begin{array}{c}\text { Locomotive } \\
\text { Velocity } \\
\left(\mathrm{ms}^{-1}\right)\end{array}$ & $\begin{array}{l}\text { Beam } \\
\text { Velocity } \\
\left(\mathrm{ms}^{-1}\right)\end{array}$ \\
\hline 1 & F $50 \mathrm{~A}$ & 21.6 & 37.2 & 0.76 & 1.2 \\
\hline 2 & $\mathrm{~F} 60 \mathrm{~A}$ & 19.0 & 21.5 & 0.67 & 0.88 \\
\hline 3 & $\mathrm{~F} 60 \mathrm{~A}$ & 14.8 & - & 1.1 & - \\
\hline 4 & F60 A & 19.0 & - & 0.76 & - \\
\hline 5 & F $60 \mathrm{~A}$ & 16.4 & - & 1.1 & - \\
\hline 6 & $\mathrm{~F} 60 / 80 \mathrm{~A}$ & 13.5 & 19.7 & 0.78 & 1.1 \\
\hline 7 & $\mathrm{~F} 80 \mathrm{~A}$ & 9.08 & 23.4 & 0.56 & 1.0 \\
\hline 8 & $\mathrm{~F} 94 \mathrm{~A}$ & 12.6 & 17.7 & 0.55 & 0.97 \\
\hline 9 & F $70 \mathrm{D}$ & 6.86 & 25.2 & 0.57 & 1.1 \\
\hline 10 & $\mathrm{C} 50 \mathrm{~A}$ & 18.5 & 19.7 & 0.90 & 1.2 \\
\hline 11 & C $50 \mathrm{~A}$ & 12.3 & 29.0 & 0.67 & 1.2 \\
\hline 12 & $\mathrm{C} 60 \mathrm{~A}$ & 17.7 & 28.2 & 0.97 & 1.4 \\
\hline 13 & $\mathrm{C} 60 \mathrm{~A}$ & 16.9 & 26.0 & 0.82 & 1.2 \\
\hline 14 & C $60 \mathrm{~A}$ & 17.1 & - & 0.81 & - \\
\hline 15 & C $60 / 80 \mathrm{~A}$ & 14.9 & 24.5 & 0.75 & 1.1 \\
\hline 16 & C $60 / 80 \mathrm{~A}$ & 13.3 & 23.5 & 0.72 & 1.1 \\
\hline 17 & C $60 / 80 \mathrm{~A}$ & 14.8 & 27.1 & 0.66 & 0.99 \\
\hline 18 & C 60/80 A & 18.5 & - & 0.71 & - \\
\hline 19 & $\mathrm{C} 80 \mathrm{~A}$ & 8.40 & 26.4 & 0.64 & 1.1 \\
\hline 20 & C $80 \mathrm{~A}$ & 8.70 & 14.5 & 0.63 & 0.87 \\
\hline 21 & No tail & 10.7 & 42.7 & 0.36 & 1.4 \\
\hline
\end{tabular}

Table 6.2 Tail comparison chart. Standard deviation values are presented where mean performance of multiple same tail tests were evaluated.

\begin{tabular}{c|c|c|c|c|c|}
$\begin{array}{c}\text { Tail } \\
\text { (Hardness } \\
\text { scale) }\end{array}$ & $\begin{array}{c}\text { Locomotive } \\
\text { acceleration } \\
\left(\mathrm{ms}^{-2}\right)\end{array}$ & $\begin{array}{c}\text { Standard } \\
\text { deviation } \\
\left(\mathrm{ms}^{-2}\right)\end{array}$ & $\begin{array}{c}\text { Locomotive } \\
\text { velocity } \\
\left(\mathrm{ms}^{-1}\right)\end{array}$ & $\begin{array}{c}\text { Standard } \\
\text { deviation } \\
\left(\mathrm{ms}^{-1}\right)\end{array}$ & $\begin{array}{c}\text { Number of } \\
\text { Tests }\end{array}$ \\
\hline F 50 A & 21.6 & - & 0.76 & - & 1 \\
\hline F 60 A & 17.3 & 2.05 & 0.90 & 0.21 & 4 \\
\hline F 60/80 A & 13.5 & - & 0.78 & - & 1 \\
\hline F 80 A & 9.08 & - & 0.56 & - & 1 \\
\hline F 94 A & 12.6 & - & 0.55 & - & 1 \\
\hline F 70 D & 6.86 & - & 0.57 & - & 1 \\
\hline C 50 A & 15.4 & 4.37 & 0.79 & 0.17 & 2 \\
\hline C 60 A & 17.2 & 0.45 & 0.87 & 0.091 & 3 \\
\hline C 60/80 A & 15.4 & 2.23 & 0.71 & 0.26 & 4 \\
\hline C 80 A & 8.55 & 0.21 & 0.64 & 0.0078 & 2 \\
\hline
\end{tabular}




\subsection{High-Speed Cinematography Performance Tests}

Limited testing was completed using high-speed digital video. Three of the higher performing tails uncovered by standard video testing were evaluated at 200 frames $\mathrm{s}^{-1}$. The higher frame rate allowed much greater detail for kinematics analysis of the mechanical fish.

Figure 6.2 displays the fish Center of Mass (COM) time trace for the 50 cut tail. Each point is separated by $5 \mathrm{~ms}$ in time, with $\mathrm{t}=0$ at the frame immediately prior to the fish firing. The scale bar is $5 \mathrm{~cm}$. The 50 cut tail trace is representative of all tests. Axes were applied to the time trace to measure the distance and position of the COM over time. The $\mathrm{x}$-axis was positioned in-line with the final lateral, or forward, motion of the fish COM. The y-axis is fit perpendicular to the $\mathrm{x}$-axis.

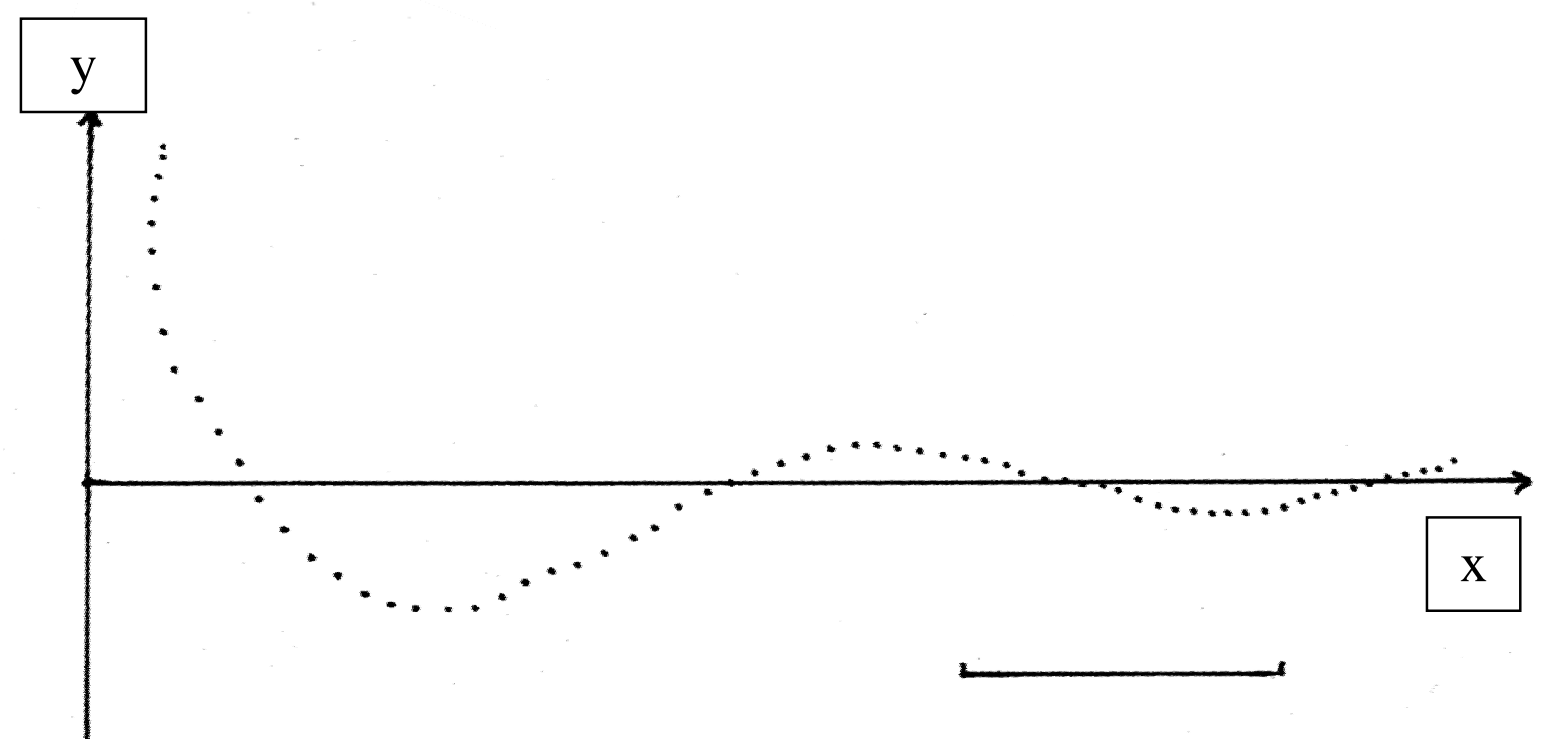

Figure 6.2 COM time trace of the 50 -cut tail. Each point is separated in time by $5 \mathrm{~ms}$. The long axis is the direction of lateral motion. Transverse motion is measured by the $y$-axis perpendicular to the $x$ axis. 


\subsubsection{Cumulative Distance}

Figure 6.3 displays the cumulative distance traveled in the $\mathrm{x}$-direction for the three fish tests. It is notable that each test begins to cover positive $\mathrm{x}$-distance (in respect to initial position) at different times. In order of increasing tail hardness material (50,60,60/80), the times to cover positive distance are $35 \mathrm{~ms}, 20-25 \mathrm{~ms}, 25-30 \mathrm{~ms}$, respectively. The 60 tail test is shortened because the fish COM point left the screen at $70 \mathrm{~ms}$. Data was still collected using a point located perpendicular to the COM, but there is more uncertainty in the measurement. Table 6.3 includes the tabular values of the COM distance covered by each fish tail.

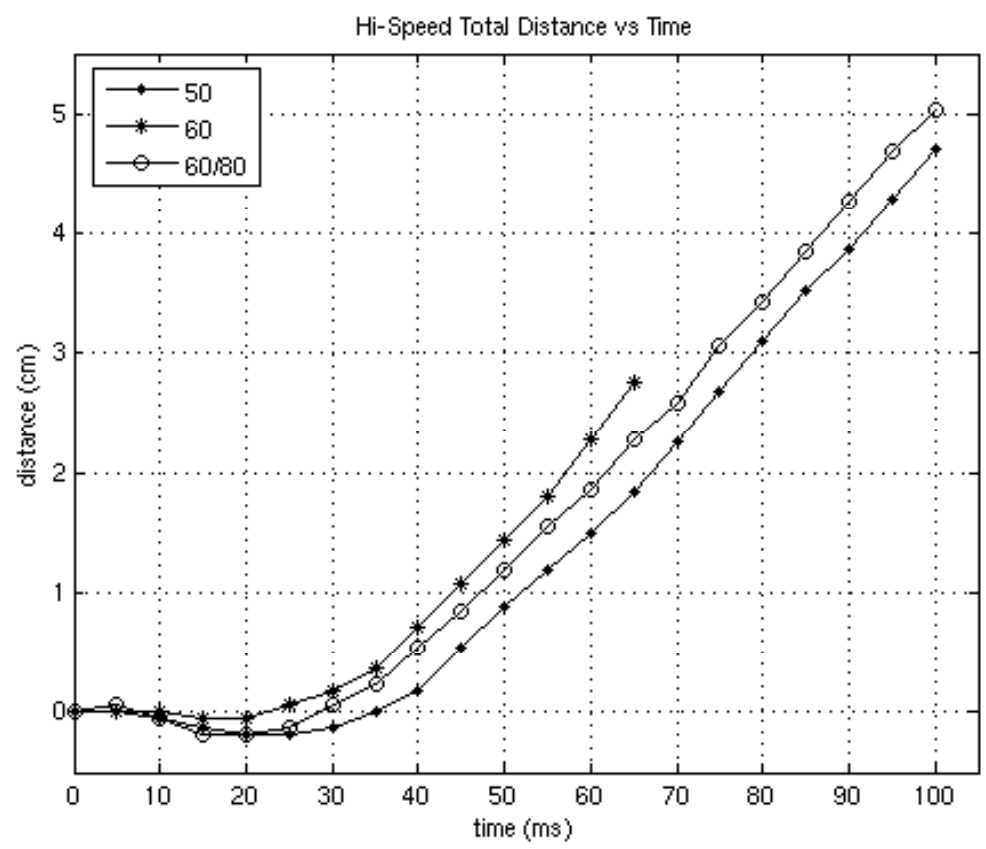

Figure 6.3 Cumulative distances over time for the three high-speed video tests. The 60 tail data stops short in the figure because of measurement uncertainty. 
Table 6.3 Cumulative distance covered by the fish COM over $100 \mathrm{~ms}$. The table includes the suspect data recorded for the 60 cut tail starting at $70 \mathrm{~ms}$.

\begin{tabular}{|c|c|c|c|}
\hline Time (ms) & 50 cut & 60 cut & $60 / 80$ cut \\
\hline 0 & 0 & 0 & 0 \\
\hline 5 & 0 & 0 & 0.6 \\
\hline 10 & -0.6 & 0 & -0.6 \\
\hline 15 & -1.2 & -0.6 & -1.8 \\
\hline 20 & -1.8 & -0.6 & -1.8 \\
\hline 25 & -1.8 & 0.6 & -1.2 \\
\hline 30 & -1.2 & 1.8 & 0.6 \\
\hline 35 & 0 & 3.6 & 2.4 \\
\hline 40 & 1.8 & 7.2 & 5.4 \\
\hline 45 & 5.373 & 10.8 & 8.4 \\
\hline 50 & 8.946 & 14.4 & 12 \\
\hline 55 & 11.924 & 18 & 15.6 \\
\hline 60 & 14.901 & 22.8 & 18.6 \\
\hline 65 & 18.474 & 27.6 & 22.8 \\
\hline 70 & 22.642 & 31.2 & 25.8 \\
\hline 75 & 26.811 & 34.8 & 30.6 \\
\hline 80 & 30.98 & 39 & 34.2 \\
\hline 85 & 35.148 & 43.2 & 38.4 \\
\hline 90 & 38.721 & 47.4 & 42.6 \\
\hline 95 & 42.89 & 51 & 46.8 \\
\hline 100 & 47.058 & 54.6 & 50.4 \\
\hline
\end{tabular}

\subsubsection{Transverse and Forward Velocities}

Raw COM velocity is presented for both forward (dx/dt) and transverse (dy/dt) directions for the 50 and 60/80-tails, figures 6.4 and 6.5, respectively. The time covers the first $100 \mathrm{~ms}$ following the fish firing. Both figures display the transverse velocity of the COM increasing rapidly with peak values occurring between 30-40 ms. Following the peak, transverse velocities gradually slow to zero at approximately $90 \mathrm{~ms}$. Forward velocity lags the transverse velocity by approximately $20 \mathrm{~ms}$. Initial forward velocities are small and either zero or reversed during the lag time. The 50-tail has a smoother lateral curve and reaches a steady velocity earlier than the combination tail. The 60-tail COM data for the transverse direction was not collected after $60 \mathrm{~ms}$ because the fish left the screen. 


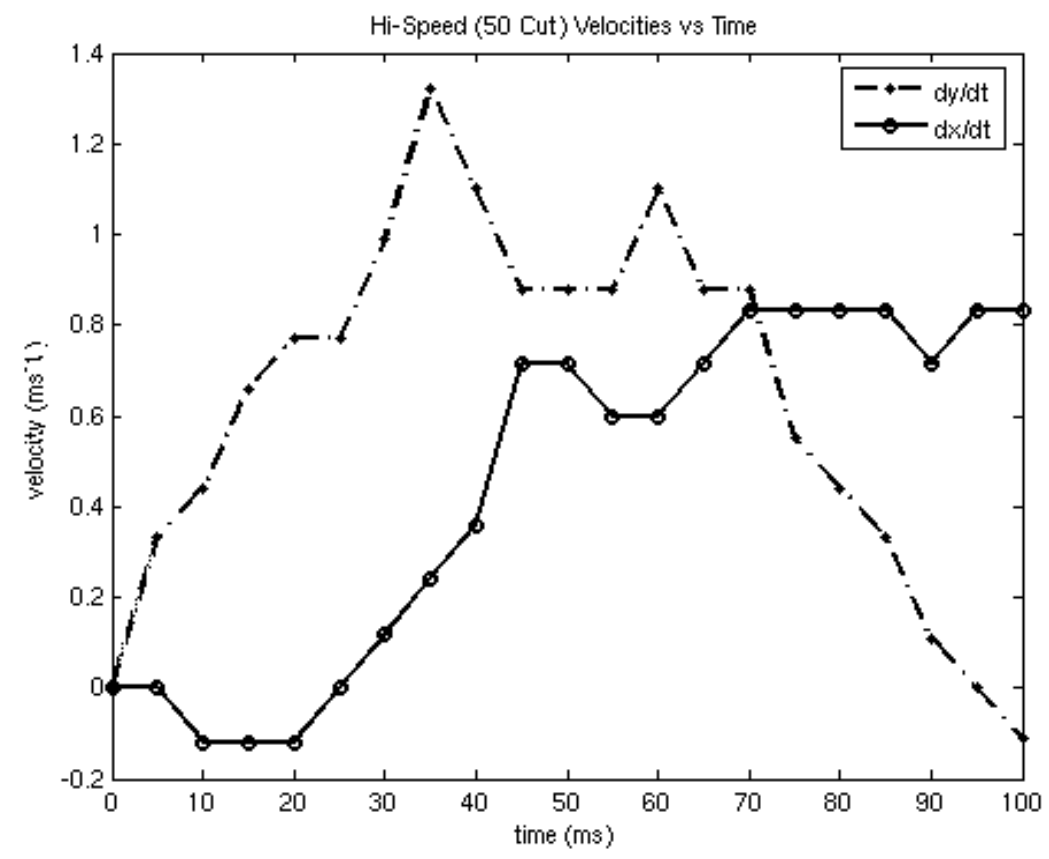

Figure 6.4 Transverse (dy/dt) and forward (dx/dt) velocity for tail 50-cut covering the first $100 \mathrm{~ms}$.

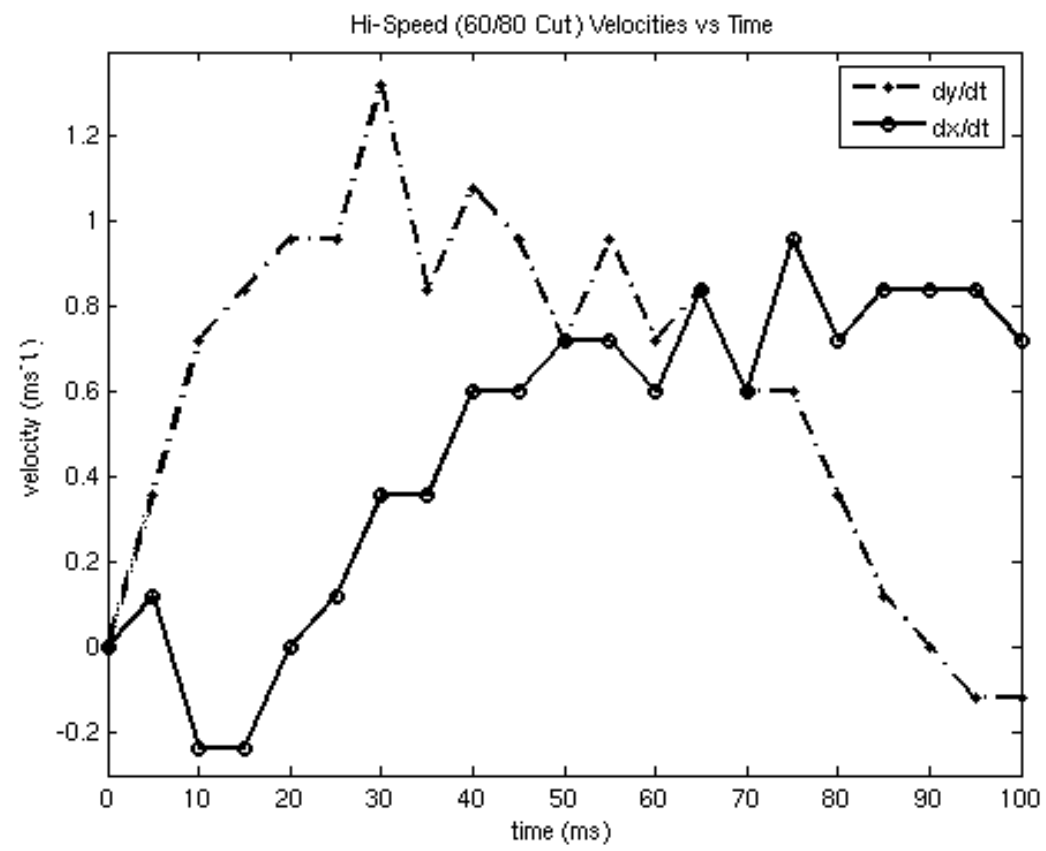

Figure 6.5 Transverse (dy/dt) and forward (dx/dt) velocity for tail 60/80-cut covering the first $100 \mathrm{~ms}$.

Further investigation of the mechanical fish raw lateral velocities is presented in the following figure. The velocity is calculated by the change in distance divided by the change in time $(\mathrm{dx} / \mathrm{dt})$. The figure shows that mean peak velocity stabilizes between $80-85 \mathrm{~ms}$ for all 
tails. This duration is consistent with the fundamental natural frequency of the mechanical fish, $11 \mathrm{~Hz}$. The value is calculated by replacing the beam length in equation (4.14) with the total fish length plus tail length. $91 \mathrm{~ms}$ is the calculated duration of the mechanical fish propulsive stroke coincident with the fundamental frequency for a free-free boundarycondition beam. The general equation for the natural frequency is:

$$
\omega_{n}=\frac{\left(\frac{(2 n+1) \cdot \pi}{2}\right)^{2}}{\left(l_{\text {fish }}+l_{\text {tail }}\right)^{2}} \cdot \sqrt{\frac{E I}{M}}
$$

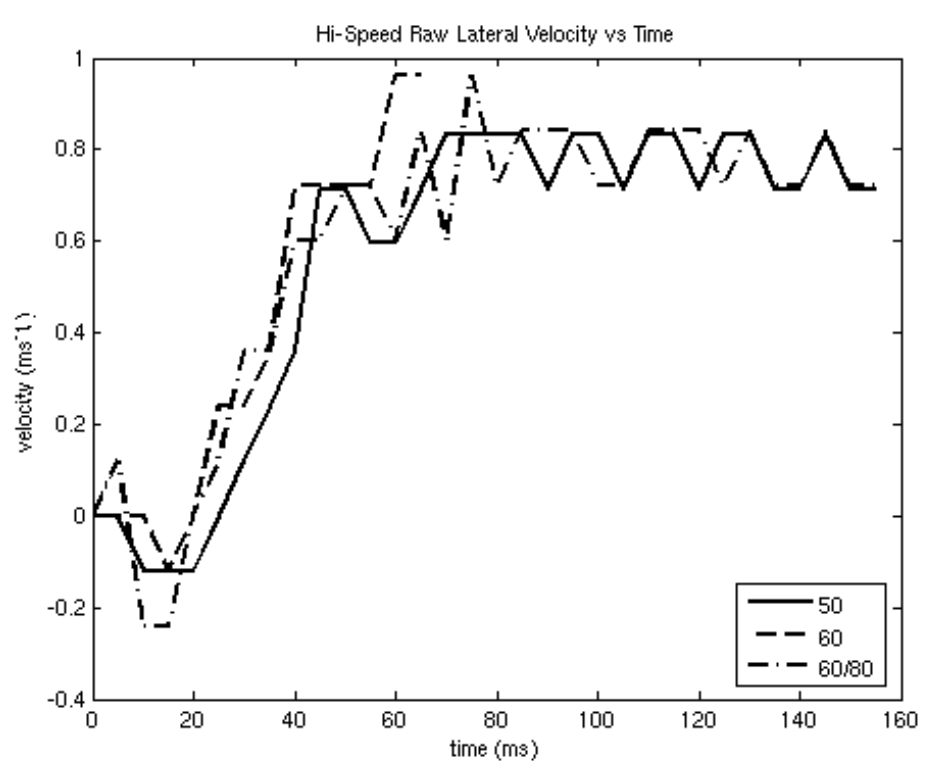

Figure 5.6 Raw forward velocities of the three high-speed tail tests over 155 ms.

The first four harmonic frequencies of the mechanical fish are 11, 31, 60, and $99 \mathrm{~Hz}$. These correspond with time durations of 91, 32, 17, and $10 \mathrm{~ms}$, respectively. These frequencies, or combinations thereof, are possibly responsible for the oscillation of the fish $\mathrm{COM}$ about the mean velocity as seen in figure 6.6 following the initial beam straightening. In figure 6.4 and 6.5, the forward and transverse velocities are correlated in anti-phase supporting this hypothesis.

The 50-tail velocity oscillates at approximately $15 \mathrm{~ms}$, peaking at $0.83 \mathrm{~ms}^{-1}$ with troughs of $0.71 \mathrm{~ms}^{-1}$. This frequency corresponds with the $3^{\text {rd }}$ natural frequency. The combination 60/80-tail velocity alternates between $0.84 \mathrm{~ms}^{-1}$ and $0.72 \mathrm{~ms}^{-1}$. It incorporates 
the $2^{\text {nd }}$ and $3^{\text {rd }}$ natural frequencies, clearly. Directly after reaching the peak mean velocity $\left(0.8 \mathrm{~ms}^{-1}\right)$, the fish oscillates at approximately $25 \mathrm{~ms}$, near the $2^{\text {nd }}$ harmonic. The fish then continues at approximately 15 ms oscillations, or the $3^{\text {rd }}$ harmonic.

The harmonic effect of the beam is also evident in the transverse direction. In figure 6.2, the COM trace shows $90 \mathrm{~ms}$ oscillation about the lateral direction of motion. This is the expected dominant frequency of oscillation and the complete propulsive stroke duration.

\subsubsection{Velocity Smoothing}

For the purpose of evaluating acceleration, smoothing the raw forward velocity is completed using a $3^{\text {rd }}$ order polynomial from the point of zero velocity, as calculated and shown in figure 6.6. Figures 6.7-6.9 display the raw data points with error bars representing position measurement error of $\pm 0.25 \mathrm{~mm}$. This position error is based on the author's measurement error. Such error corresponds to $\pm 0.06 \mathrm{~ms}^{-1}$ velocity measurement error bars. The polynomials are plotted in the figures, and the equations are provided:

$$
\begin{aligned}
& v_{50}=2319 \cdot t^{3}-506.18 \cdot t^{2}+35.505 \cdot t \\
& v_{60}=3740.4 \cdot t^{3}-713.39 \cdot t^{2}+42.937 \cdot t \\
& v_{60 / 80}=2544.5 \cdot t^{3}-524.92 \cdot t^{2}+35.542 \cdot t
\end{aligned}
$$

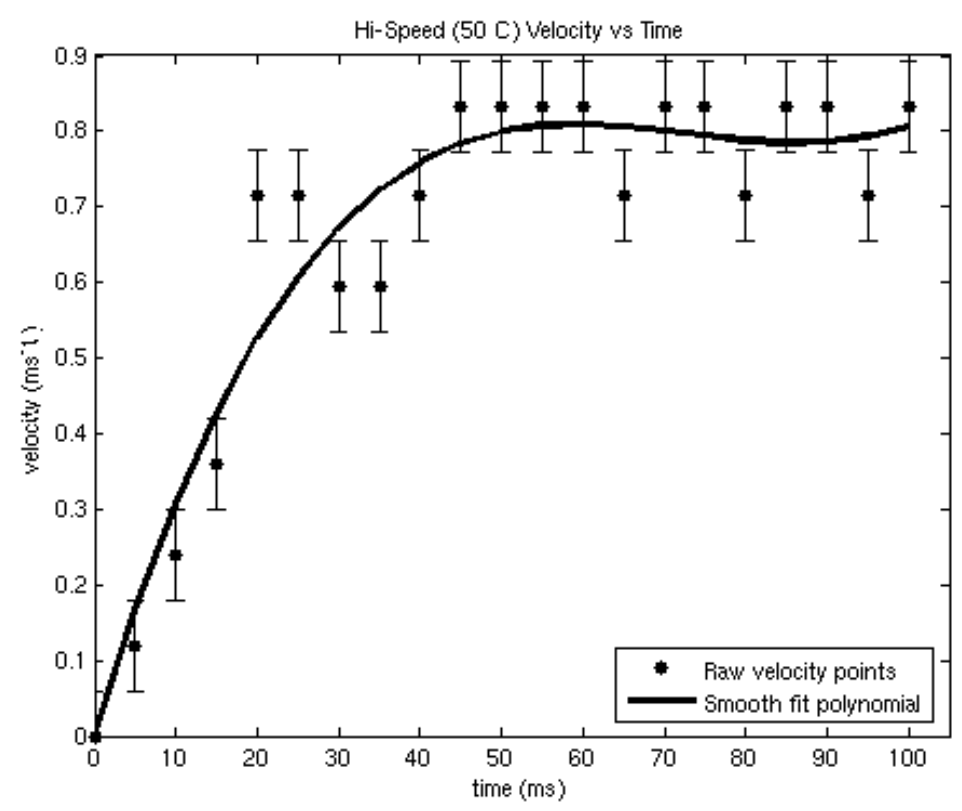

Figure 6.7 50-tail smoothed velocity. 


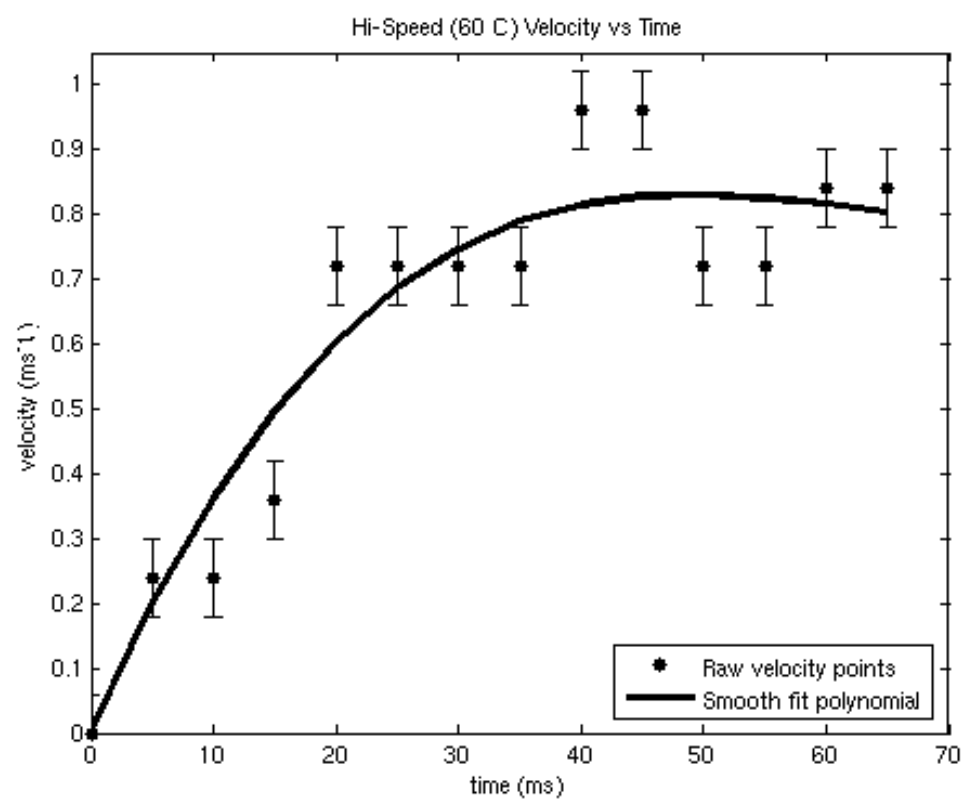

Figure 6.8 60_tail smoothed velocity.

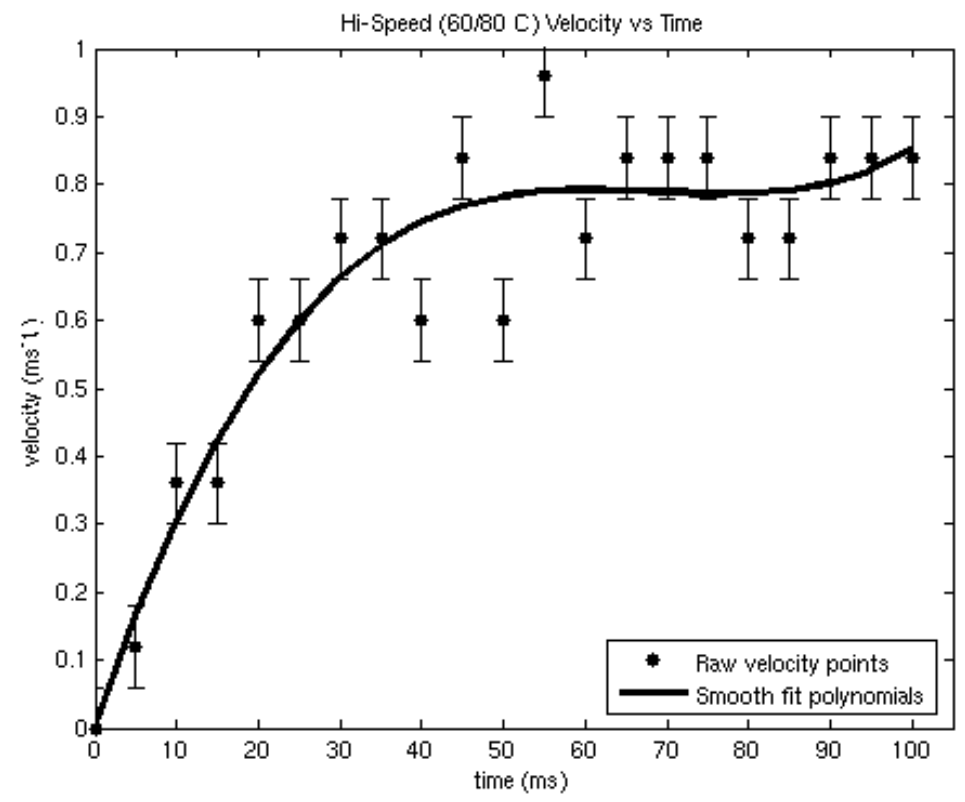

Figure 6.9 60/80-tail smoothed velocity.

The low-order polynomial fits are not able to capture the high-frequency motions discussed in section 6.4.2. It is expected that the velocity smoothing will underestimate the accelerations of the high-frequency fast-start maneuver. 


\subsubsection{Acceleration}

Calculating acceleration is completed using two methods. One method uses the cumulative COM distance over time from figure 6.3 with the equation for projectile motion. The other method uses the derivative of the smoothed velocity polynomials from figures 6.6-6.8.

From zero velocity the equation of projectile motion simplifies to:

$$
\vec{x}=\frac{1}{2} \vec{a} \cdot t^{2}
$$

where $\vec{X}$ is the cumulative distance and $\vec{a}$ is the rate of acceleration. Points of zero velocity are calculated and displayed in figure 6.6. The point of zero velocity is the position of zero time for acceleration calculations. Figure 6.10 displays the maximum acceleration rate versus time for a confident distance of measure. The thick dashed line and associated dots are the calculated accelerations using the cumulative distance to the specified time on the $\mathrm{x}$-axis (from figure 6.3). The thin-solid lines surrounding the dashed line represent error bounds of $1 / 4 \mathrm{~mm}$ distance on either side.

Figure 6.10 shows that increasing the cumulative time-distance data decreases the probable measurement error while also decreasing the calculated maximum performance. The maximum acceleration rate of $48 \mathrm{~ms}^{-2}$ for both 50 and 60/80-tails occurs over the film rate of $5 \mathrm{~ms}$. A more conservative value for maximum acceleration is approximately $36 \mathrm{~ms}^{-2}$, bounded by possible error between $34-38 \mathrm{~ms}^{-2}$. This value is calculated using cumulative distance over $20 \mathrm{~ms}$ for both tails. A true maximum acceleration is difficult to calculate with cinematography, even at 200 frames s ${ }^{-1}$. 

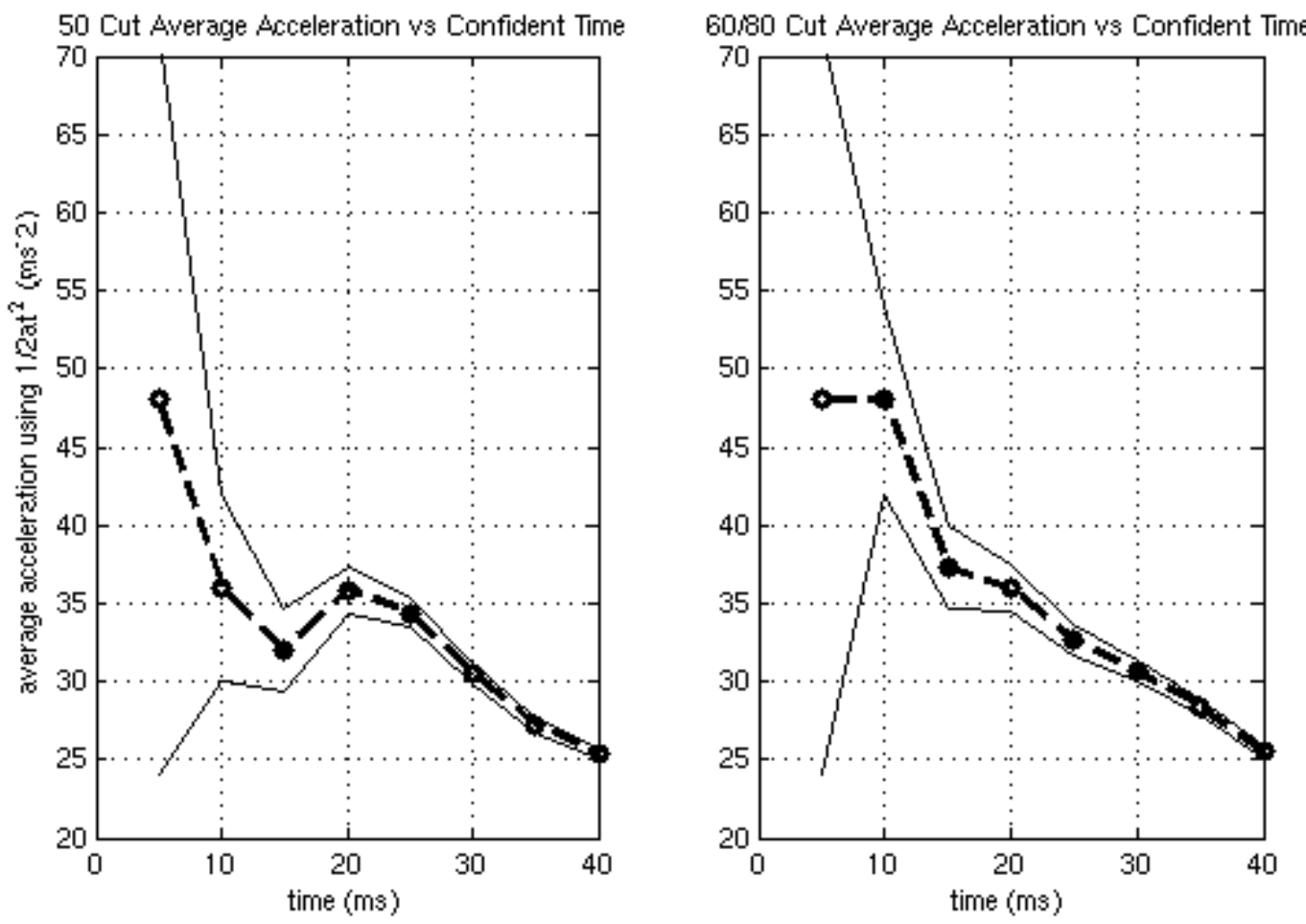

Figure 6.10 Maximum acceleration rate using the equation for projectile motion and a confident distance measurement in time, from figure 6.3.

As an additional check, the smoothed velocity polynomials are used to calculate maximum acceleration. This method dampens the high frequency noise associated with frame-by-frame measurements of distance, therefore providing a clean derivative for acceleration. The equations of the polynomials displayed in figure 6.11 are:

$$
\begin{aligned}
& a_{50}=6957 \cdot t^{2}-1012.36 \cdot t+35.505 \\
& a_{60}=11221.2 \cdot t^{2}-1426.78 \cdot t+42.937 \\
& a_{60 / 80}=7633.5 \cdot t^{2}-1049.84 \cdot t+35.542
\end{aligned}
$$

Maximum acceleration values estimated at $5 \mathrm{~ms}$ for the 50 and 60/80-tails were approximately $30 \mathrm{~ms}^{-2}$, and $36 \mathrm{~ms}^{-2}$ for the 60 -tail. For comparison to the previous method, all of the tails were between 17.5 and $19 \mathrm{~ms}^{-2}$ at $20 \mathrm{~ms}$. 


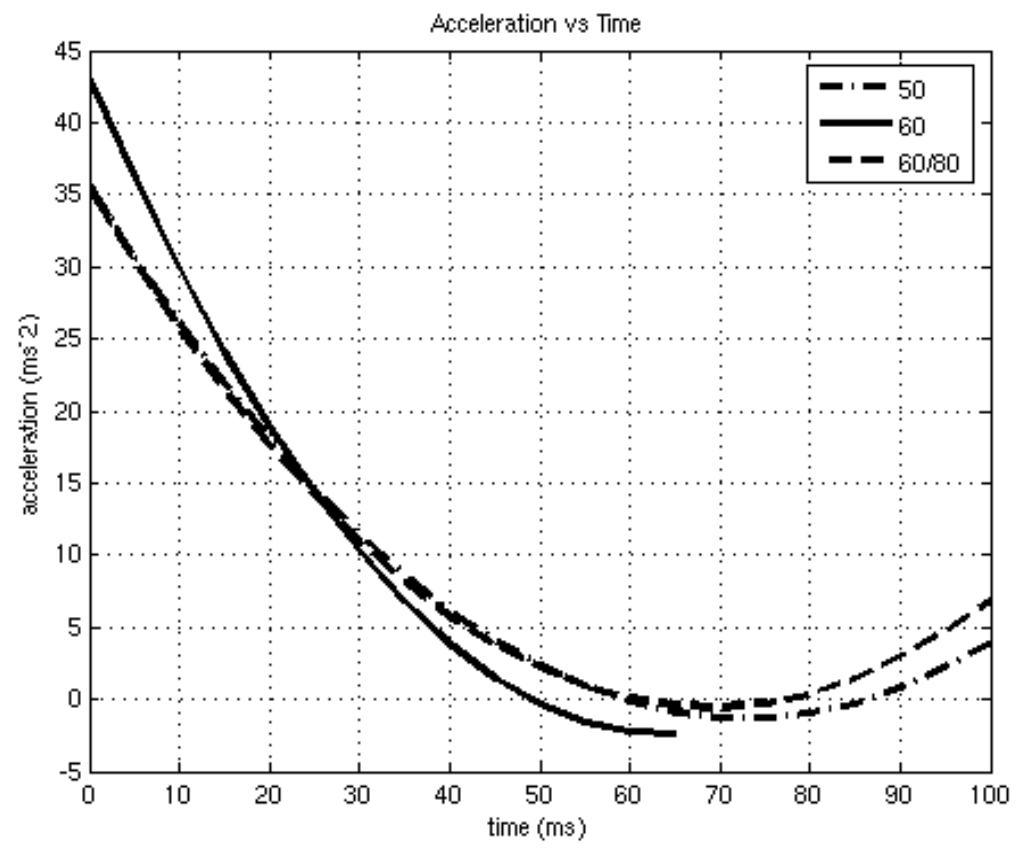

Figure 6.11 Acceleration plot using the derivative of the smoothed velocities plots for the three tails.

Table 6.4 displays the maximum and mean maximum performance parameters of the mechanical fast-start fish, calculated from the 200 frames $\mathrm{s}^{-1}$ data.

Table 6.4 Maximum performance parameters derived from the time-distance data at $200 \mathrm{~Hz}$. Method 1 are maximum values from raw data, while method 2 are smoothed values. 60/80 maximum velocity is given at the peak before the first dip in the polynomial (figure 6.9).

\begin{tabular}{|c|c|c|c|c|c|}
\hline Run & $\begin{array}{c}\text { Tail } \\
\text { (Hardness } \\
\text { scale) }\end{array}$ & $\begin{array}{c}\text { Method 1 } \\
\text { Maximum } \\
\text { Acceleration } \\
\text { (ms-2) }\end{array}$ & $\begin{array}{c}\text { Method 2 } \\
\text { Maximum } \\
\text { Acceleration } \\
\text { (ms-2) }\end{array}$ & $\begin{array}{c}\text { Method 1 } \\
\text { Maximum } \\
\text { Velocity } \\
\text { (ms-1) }\end{array}$ & $\begin{array}{c}\text { Method 2 } \\
\text { Maximum } \\
\text { Velocity } \\
\text { (ms-1) }\end{array}$ \\
\hline 22 & C 50 A & 48 & 30 & 0.83 & 0.81 \\
\hline 23 & C 60 A & - & 36 & 0.96 & 0.83 \\
\hline 24 & C 60/80 A & 48 & 30 & 0.96 & 0.79 \\
\hline
\end{tabular}

\subsubsection{Hydrodynamic Efficiency}

The fish fast-start is not a highly efficient swimming event [4.2.1.1]. The efficiency estimate is based on the transfer of energy from elastic potential to kinetic swimming energy. The fish beam placed in $23 \mathrm{~cm}$ curvature retains 3.9 Joules of energy. An error of $1 \mathrm{~cm}$ in either direction produces 3.6-4.3 Joules of stored energy. When fired, the fish mass of $1.1 \mathrm{~kg}$ accelerates to a mean velocity of $0.8 \mathrm{~ms}^{-1}$. The energy transferred to kinetic energy is approximately 0.42 Joules. The efficiency is approximately $11 \%$, calculated by $E_{p} / E_{k}$. 
Assuming the above error, the efficiency is between $10-12 \%$. The fast-start efficiency for real fish is between 16-39\% (Frith and Blake, 1995).

\subsubsection{Vortex Wake Patterns}

\subsubsection{Double Vortex Pattern}

Figure 6.12 shows a progressive fast-start fish wake using the combination $60 / 80$ cut-tail at 66 ms intervals. This sequence shows the initial propulsive vortex shed at approximately $50^{\circ}$ from the axis of lateral motion. The vortex is shed at approximately $100 \mathrm{~ms}$ after the fish fires. The second shed vortex from the fish tail fin is released at approximately $60^{\circ}$ off the $x-$ axis in the opposite direction. The point where the vortices are shed is nearly coincident.

The time for this second vortex to shed is approximately $200 \mathrm{~ms}$ from the fish firing. The duration between firing and shed vortices corresponds with the $1^{\text {st }}$ natural frequency of the fish. Qualitatively, the first vortex moves at approximately twice the speed as the second. It also appears that the harmonic oscillations of the fish after reaching the mean peak velocity continue to fuel the second vortex induced propulsive jet for a short time. Further images, not displayed here, show the steady progression of the second created vortex along the $60^{\circ}$ path and the first vortex crashing into the side of the tank. 


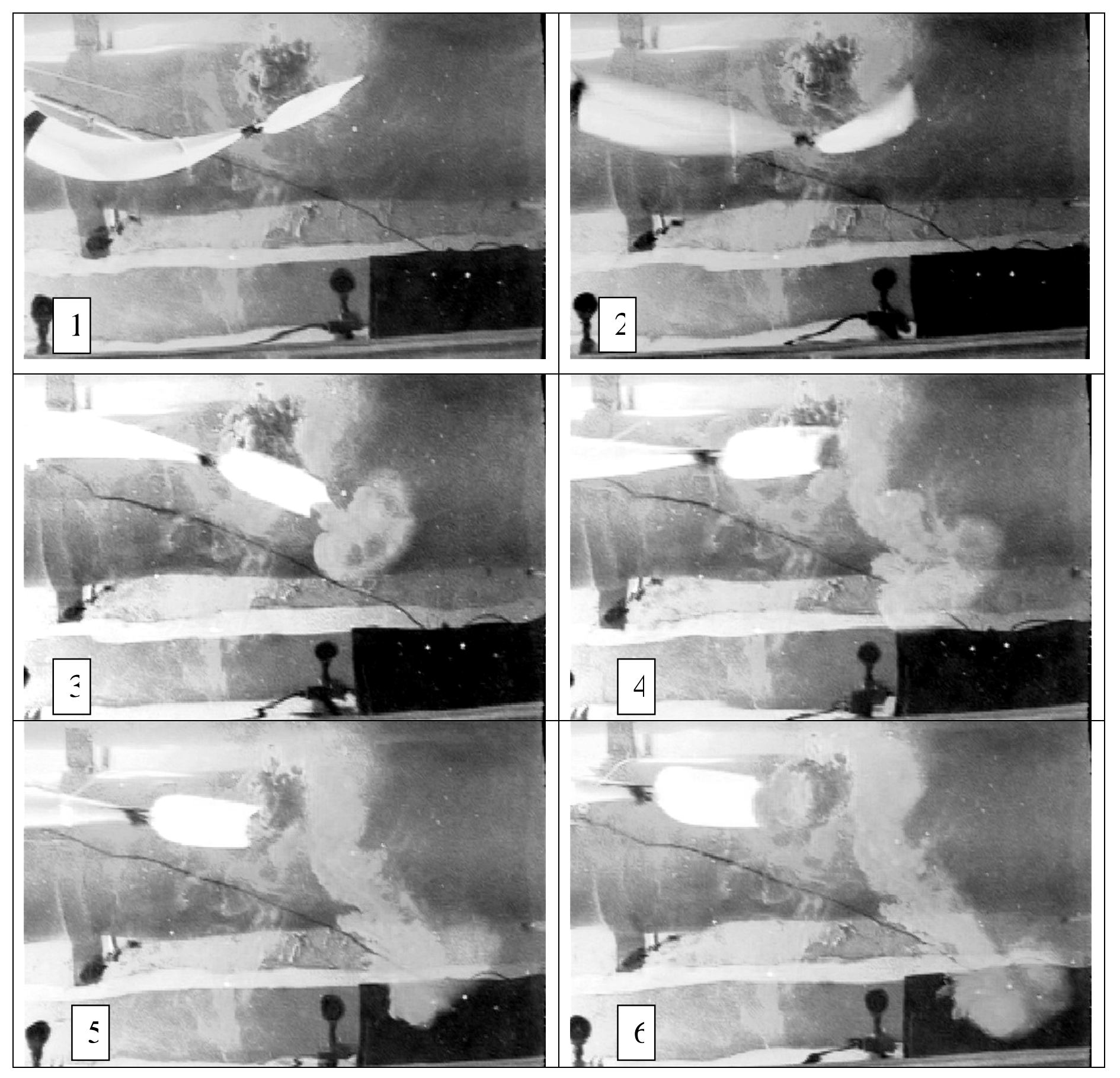




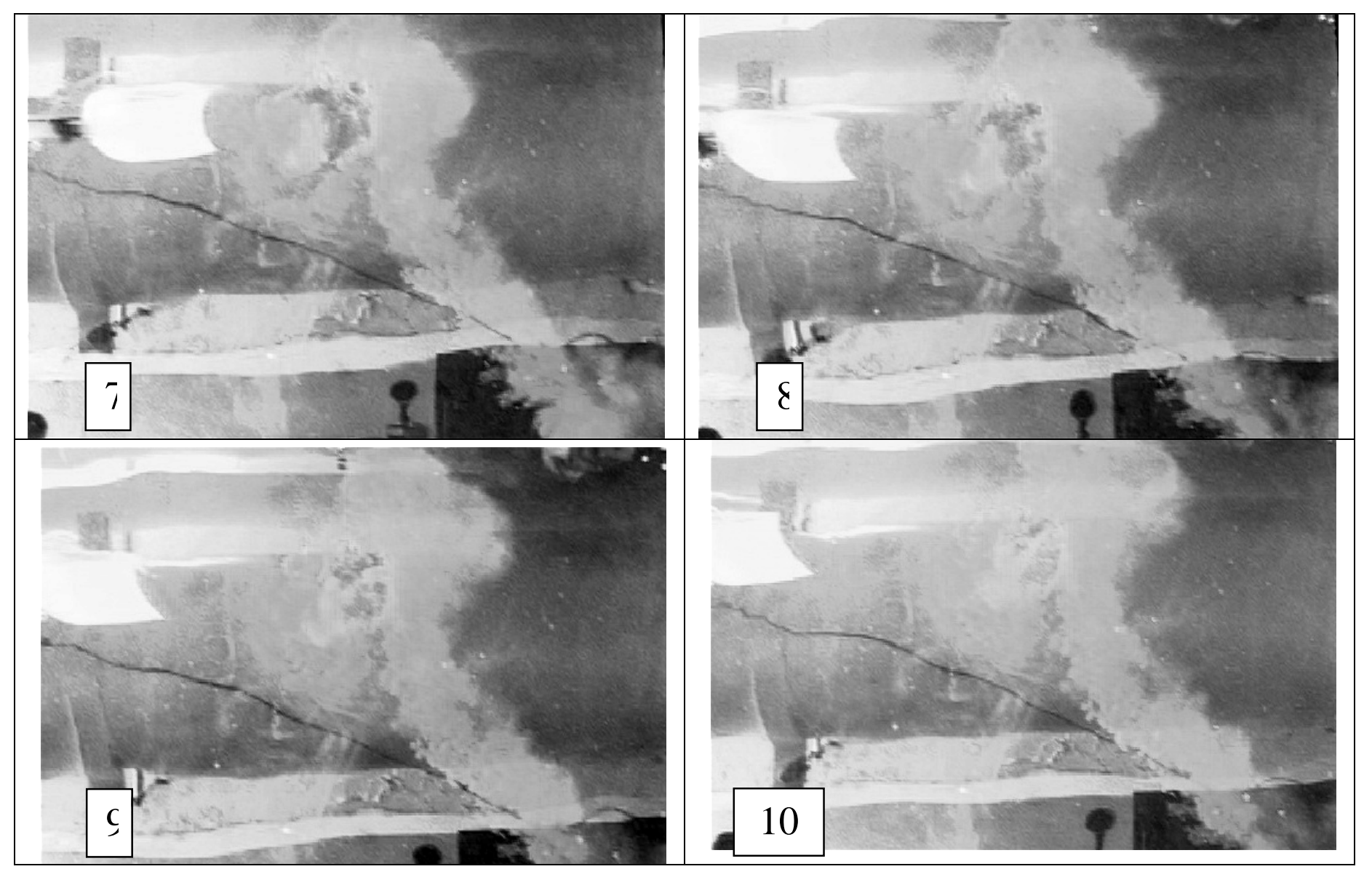

Figure 6.12 60/80 cut tail displaying the double vortex wake at $66 \mathrm{~ms}$ separation between frames. The second vortex is first noticed between in frame 4 (the frame between 3 and 4 is the first recognized formation, not pictured).

The figure 6.13 sequence of the 60 cut-tail shed vortices includes an initial vortex that quickly accelerates from the propulsive stroke at approximately $50^{\circ}$ from the $x$-axis. The time between images is $66 \mathrm{~ms}$. The second shed vortex was released at approximately $70^{\circ}$. The two vortices pattern of vortex creation was the standard pattern noticed during tail testing; approximately occurring three out of every four tests. 


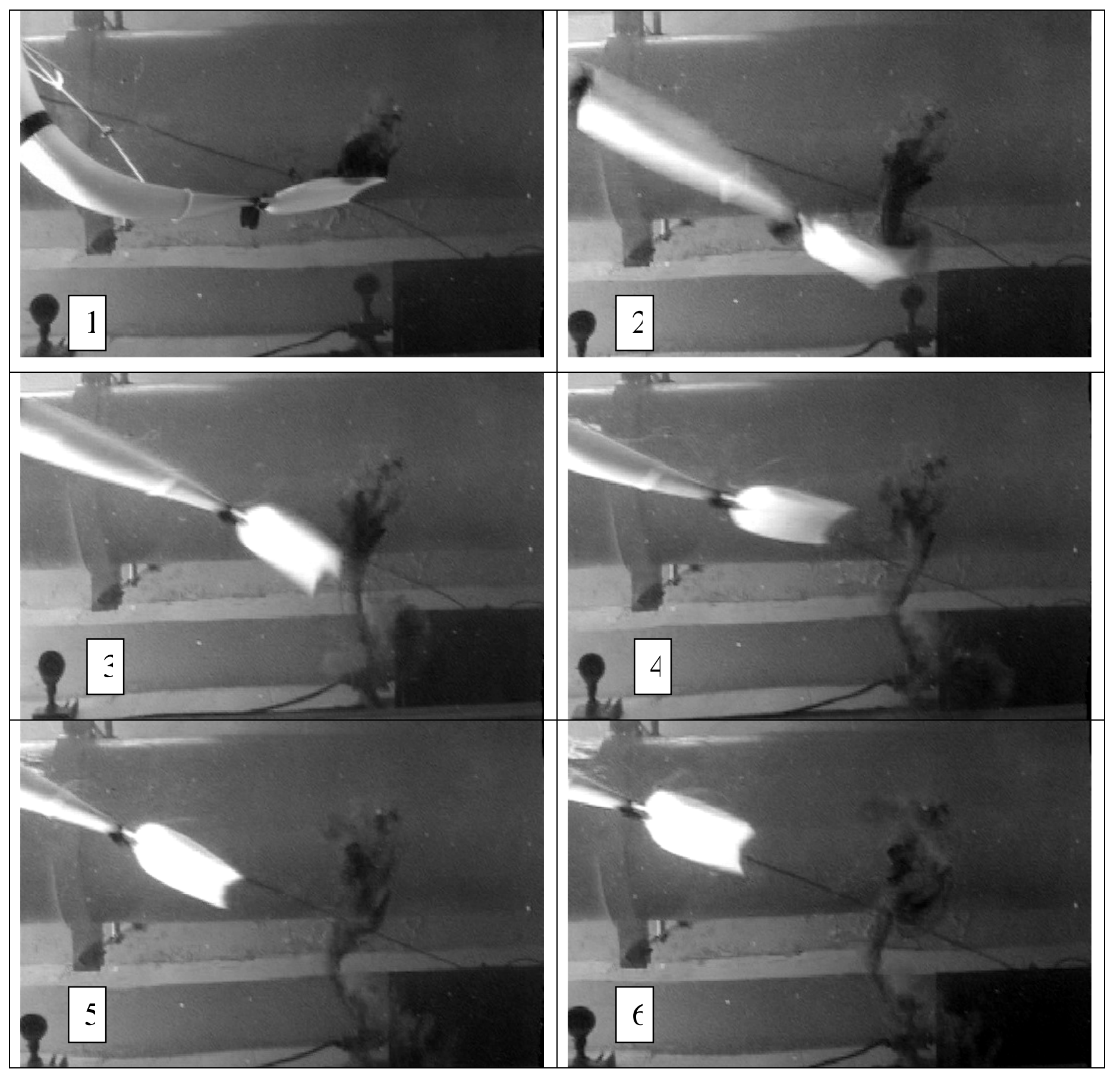




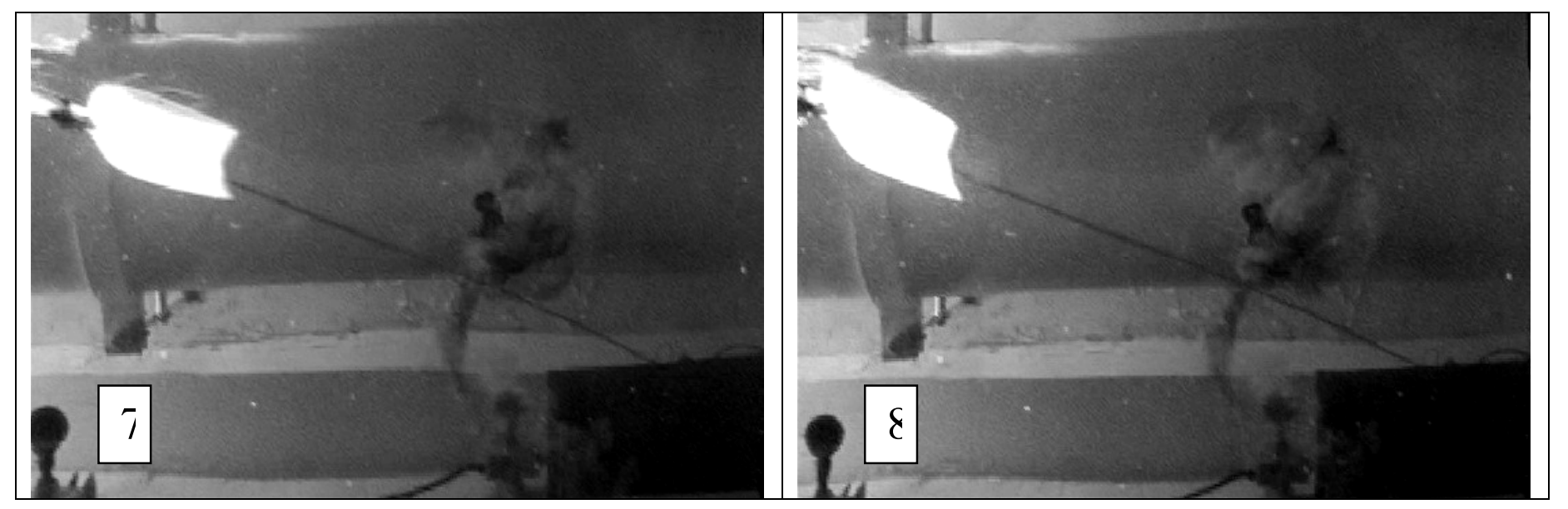

Figure 6.1360 cut tail displays the double vortex wake at $66 \mathrm{~ms}$ separation between frames.

\subsubsection{Single Vortex Pattern}

The single vortex pattern was observed in approximately one out of four tail tests. This faststart produces a single propulsive vortex shed into the wake from the cut 60 -tail. The vortex induces a second in the wake and a strong momentum jet continues at approximately $35^{\circ}$ off the axis of lateral motion. The images are $33 \mathrm{~ms}$ apart in figure 6.14. 


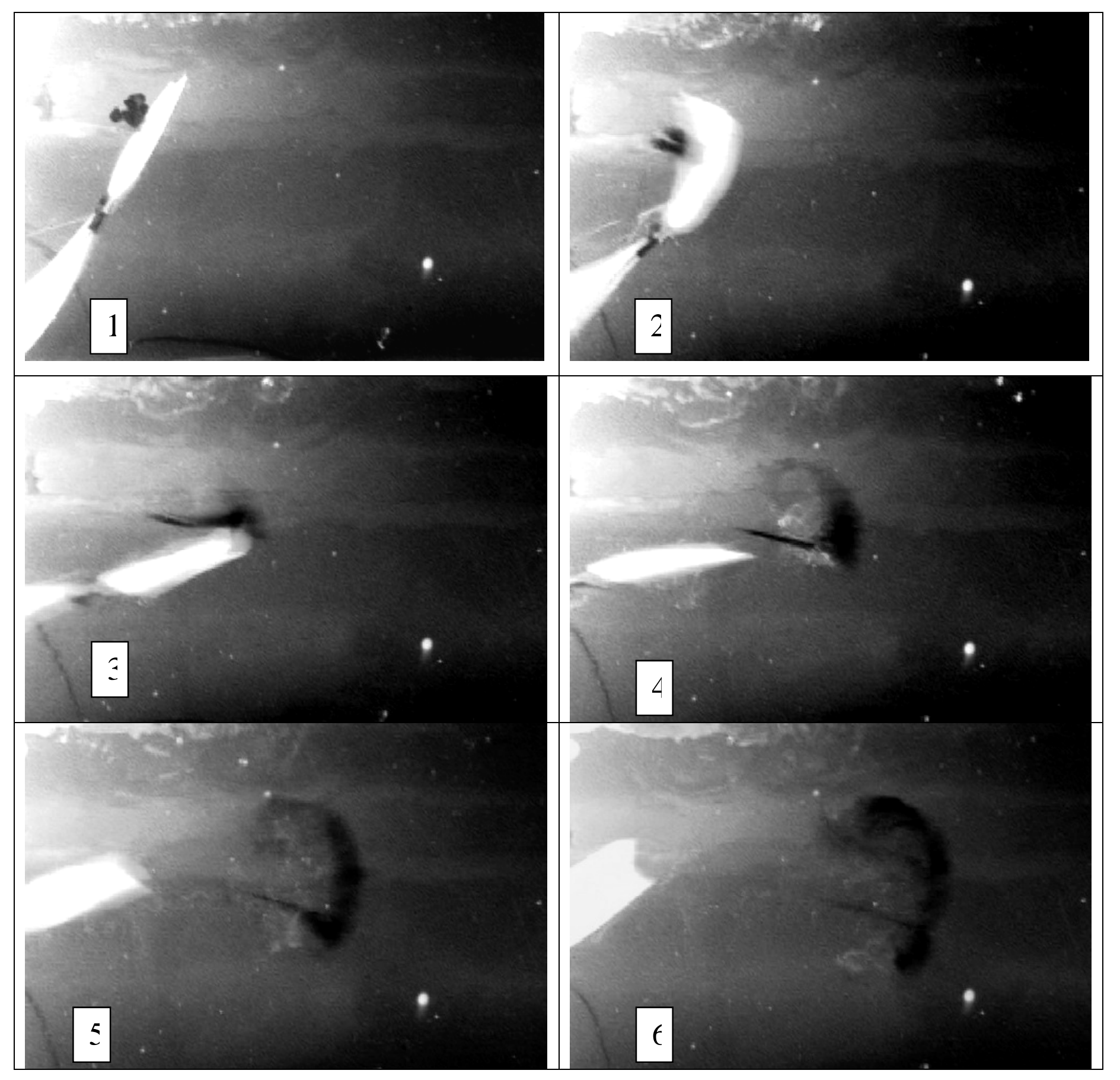




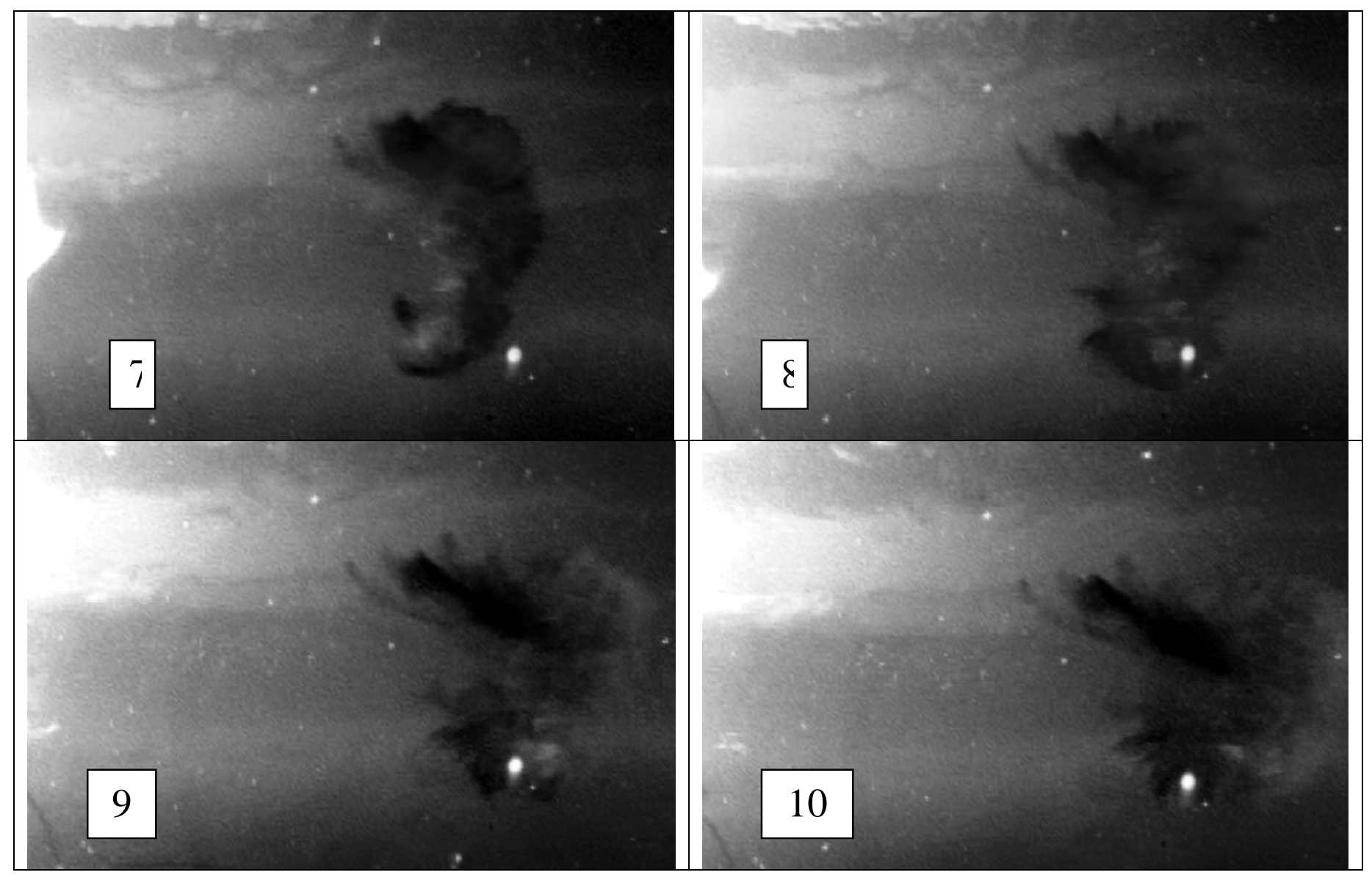

Figure 6.1460 cut tail displays the single vortex wake at $33 \mathrm{~ms}$ separation between frames.

No quantitative results are available to compare the possible efficiency advantage of one vortex pattern over the other. Qualitatively, the single vortex pattern produced the strongest momentum jet directed opposite the direction of desired motion. Water was observed splashing out of the rear of the tank when this pattern occurred. However, combining the two vortices pattern may produce the same, or more, momentum directed opposite forward motion. Real fish fast-start vortex patterns were not readily available for comparison, but a vortex patterns from a simulated fast-start apparatus are (Ahlborn et al., 1997).

The apparatus included a preparatory phase and propulsive phase. The paper presented the flow field around the simulated fish tail, visualized using aluminum seeding of the water surface. Looking only at the propulsive phase of the fast-start, the simulation vortex pattern matches the single vortex pattern described above. A single vortex is created that induces another in the vortex in the surrounding fluid, creating a propulsive momentum jet. 
This suggests that the single vortex pattern in this thesis may best represent the vortex wake of a real fish. 


\section{Chapter 7}

\section{Future Work and Conclusion}

\subsection{Future Work}

\subsubsection{Including the Preparatory Stroke}

In this thesis, the preparatory stroke was not included, in order to simplify the mechanical design. However, doing so also limited the maximal expected performance of the fish. Future work to include the preparatory stroke with the current mechanical fish product has great potential to closely match real fish performance. Ahlborn and others (1997) describe a fast-start propulsion mechanism termed the reversal of momentum.

The reversal of momentum is described by an initial angular momentum imparted to the fluid by the fish coiling, producing a shed vortex. The tail imparts a reversed angular momentum as it uncoils, the propulsive stroke vortex. The interaction of the opposite signed vortices momentarily stops the fluid, which then acts like a 'stepping stone' that the fish pushes against for forward propulsion.

The equation of forward thrust, $F_{x}$, as a function of the angular momentums imparted to the fluid is given by (Ahlborn and others, 1997):

$$
F_{x}=-\frac{1}{y_{2}} \frac{G_{f}-G_{i}}{\Delta t}
$$

$y_{2}$ is the moment arm of force which stops or reverses the first vortex, initial and final momenta are $G_{i}$ and $G_{f}$, respectively, and $\Delta t$ is the duration of applied torque. The two momentum extremes of the equation are a 'stealth' swimming mode with $G_{f}=0$ with no vortex 
left in the wake, or $G_{i}=0$ with no pre-existing vortex in the wake. It is easily seen that the existence of an equal and opposite initial vortex created in the propulsive stroke could improve thrust performance by $50 \%$.

\subsubsection{Fin Form and Position}

The importance of a large surface area at the caudal region of the mechanical fish is important for high acceleration and thrust production (Webb 1978). The larger surface area accelerates a greater amount of water, therefore producing a greater momentum change (Frith and Blake, 1991). Better estimates of the scaled surface area of the propulsive fins may improve the mechanical fish performance.

Frith and Blake (1991) provide aspect ratio (AR) values for the three pike propulsive fins, 1.53, 1.27, and 1.83 for the anal, dorsal, and caudal fin, respectively. Applying the AR values, and accurate surface areas of all fins separately should improve performance. The dorsal and anal fins were noted by Frith and Blake (1991) to flex into greater angles of attack relative to the fish forward motion. The bending increases the useful direction of thrust, but decreases the overall value. The positioning of the anal and dorsal fins contributes to the faststart thrust before the caudal fin, therefore allowing acceleration to begin earlier. This is another reason for inclusion of separate anal and dorsal fins; the delay of forward distance covered could be improved.

\subsubsection{Neutral and Un-Tethered Mechanical Fish}

The fish performance may also be affected by the tethered nature of this thesis experimental set-up. Maximum velocity in the lateral direction may be larger than reported because of the transverse pull of the tethered line connecting the fish. It was assumed that the small timedistance data of interest would be unaffected, which may not be completely accurate. Also, the air line contributes to the added mass of the fish motion. A neutrally buoyant, un-tethered mechanical fish would not include these added error-producing effects. 


\subsubsection{Use of Accelerometers}

Accelerometers placed at different positions of the mechanical fish, including the COM, would be a step closer to instantaneous performance values. This would allow better comparison with the Harper and Blake $(1990,1991)$ papers on northern pike performance.

\subsection{Conclusion}

In this thesis, a mechanical fast-start fish was designed and fabricated to mimic the propulsive stage of a startle response fast-start. The mechanical fish achieved maximum accelerations of $48 \mathrm{~ms}^{-2}$ with forward velocity peaks near $1 \mathrm{~ms}^{-1}$. The acceleration rate is right inline with earlier studies using cinematography for performance calculations. Weihs (1973) measured acceleration values of an unspecified pike species at $50 \mathrm{~ms}^{-2}$. However, more recent investigations have proved much greater start-up accelerations are achieved by the fast-start specialist species. For example, Harper and Blake (1991) calculated mean maximum values at $120 \mathrm{~ms}^{-2}$.

Nevertheless, the calculated values of velocity, acceleration, and hydrodynamic efficiency (11\%) of the mechanical fish are encouraging. This thesis provided a basis for further investigation into the fast-start maneuver from an engineering point of view. Continued development with this model, and possible implementation of the energy beam technique on a free-swimming autonomous vehicle may someday allow a greater measure of safety in unpredictable aquatic regions. 


\section{Bibliography}

Ahlborn, B., Chapman, S., Stafford, R., Blake, R. W. and Harper, D. G. (1997). Experimental simulation of the thrust phases of fast-start swimming of fish. Journal of Experimental Biology, 200, 2301-2312.

Blake, R. W. (2004). Review paper: Fish functional design and swimming performance. Journal of Fish Biology, 65, 1193-1222.

Domenici, P. D. and Blake, R. W. (1991). The kinematics and performance of the escape response in the angelfish (Pterophyllum eimekei). Journal of Experimental Biology, 156, 187-205.

Domenici, P. D. and Blake, R. W. (1997). The kinematics and performance of fish fast-start swimming. Journal of Experimental Biology, 200, 1165-1178.

Frith, H. R. and Blake, R. W. (1991). Mechanics of the startle response in the northern pike, Esox lucius. Canadian Jouranal of Zoology, 69, 2831-2839.

Frith, H. R. and Blake, R. W. (1995). The mechanical power output and Hydromechanical efficiency of northern pike (Esox lucius) fast-starts. Journal of Experimental Biology, 198, 1863-1873.

Hale, M. E. (2002). S- and C-start escape responses of the muskellunge (Esox masquinongy) require alternative neuromotor mechanisms. Journal of Experimental Biology, 205, 2005-2016.

Harper, D. G. and Blake, R. W. (1989). Short communication: A critical analysis of the use of high-speed film to determine maximum accelerations of fish. Journal of Experimental Biology, 142, 465-471.

Harper, D. G. and Blake, R. W. (1990). Fast-start performance of rainbow trout Salmo gairneri and northern pike Esox lucius. Journal of Experimental Biology, 150, 321342.

Harper, D. G. and Blake, R. W. (1991). Prey capture and the fast-start performance of northern pike, Esox lucius. Journal of Experimental Biology, 155, 175-192.

Rand, D. M. and Lauder, G. V. (1981). Prey capture in the chain pickerel, Esox niger: correlations between feeding and locomotor behavior. Canadian Jouranal of Zoology, 59, 1072-1078.

Schriefer, J. E. and Hale, M. E. (2004). Strikes and startles of northern pike (Esox lucius): a comparison of muscle activity and kinematics between S-start behaviors. Journal of Experimental Biology, 207, 535-544. 
Triantafyllou, M. S. and Triantafyllou, G. S. (1995). An efficient swimming machine. Scientific American, 272, 64-70.

Triantafyllou, M. S., Triantafyllou, G. S. and Yue, D. P. (2000). Hydrodynamics of fishlike swimming. Annual Review of Fluid Mechanics, 32, 33-53.

Videler, J. J. (1993). Fish Swimming. London: Chapman and Hall.

Wakeling, J. M. (2001). Biomechanics of fast-start swimming in fish. Comparative Biochemistry and Physiology, 131, 31-40.

Webb, P. W. (1975). Acceleration performance of rainbow trout Salmo gairdneri and green sunfish Lepomis cyanellus. Journal of experimental biology, 63, 451-465.

Webb, P. W. (1976). The effect of size on the fast-start performance of rainbow trout Salmo gairdneri and a consideration of piscivorous predator-prey interactions. Journal of Experimental Biology, 65, 157-177.

Webb, P. W. (1977). Effects of median-fin amputation on fast-start performance of rainbow trout (Salmo gairdneri). Journal of Experimental Biology, 68, 123-135.

Webb, P. W. (1984). Body form, locomotion and foraging in aquatic vertebrates. American Zoologist, 24, 107-120. 